\title{
Two stochastic processes shape diverse senescence patterns in a single-cell organism
}

Short title: Stochastic processes shape senescence

\author{
Ulrich K. Steiner*1,2,3,4 , Adam Lenart ${ }^{1 \ddagger}$, Ming Ni ${ }^{3,4,5 \ddagger}$, Peipei Chen ${ }^{3,6}$, Xiaohu Song ${ }^{3}$, François \\ Taddei $^{3,4}$, Ariel B. Lindner ${ }^{3,4 \dagger}$, \& James W. Vaupel ${ }^{1 \dagger}$
}

1

$2 \quad{ }^{1}$ Center on Population Dynamics, Campusvej 55, 5230 Odense, Denmark

$3 \quad{ }^{2}$ Biology Department, University of Southern Denmark, Campusvej 55, 5230 Odense, Denmark

$4 \quad{ }^{3}$ Center for Research and Interdisciplinarity, Paris Descartes University, 75014, Paris, France

$5 \quad{ }^{4}$ INSERM U1001, 75014 Paris, France

$6 \quad{ }^{5}$ Current address: BGI Shenzhen, Shenzhen, China

$7 \quad{ }^{6}$ Current address: National Center for Nanoscience and Technology, Beijing, China

8

9

\section{*Corresponding author: usteiner@biology.sdu.dk}

$11{ }^{\dagger}$ Joint senior authors

$12{ }^{\ddagger}$ Equal contribution of second authors

13 Keywords: senescence, mechanisms of aging, evolution of aging, asymmetry, damage accumulation, 14 aging models,

15 Authors contributions: UKS designed the study, UKS, MN \& PC performed the experiments, UKS, 16 AL, XS analyzed the data, all authors substantially contributed to discussions and writing the 17 manuscript, UKS wrote the first and final draft of the manuscript. 


\section{Abstract}

Despite advances in aging research, a multitude of aging models, and empirical evidence for diverse senescence patterns, understanding is lacking of the biological processes that shape senescence, both for simple and complex organisms. We show that for a isogenic Escherichia coli bacterial population senescence results from two stochastic processes. A primary random deterioration process within the cell, such as generated by random accumulation of damage, leads to an exponential increase in mortality early in life followed by a late age mortality plateau; a secondary process of stochastic asymmetric transmission of an unknown factor at cell fission influences mortality. This second process is required to explain the difference between the classical mortality plateaus detected for young mothers’ offspring and the near non-senescence of old mothers' offspring as well as the lack of a mother offspring correlation in age at death. We observed that life span is predominantly determined by underlying stochastic stage dynamics. Our findings support models based on stage-specific actions of alleles for the evolution of senescence. This support might be surprising since these models that have not specifically been developed in the context of simple, single cell organisms. We call for exploration of similar stochastic influences beyond simple organisms.

\section{Introduction}

One of the major challenges for biodemographic research on aging is to understand what drives senescence patterns (Vaupel et al. 1998, López-Otín et al. 2013). This challenge is illustrated by the variety of aging models that assume different, as yet unverified generating processes (Hamilton 1966, Kirkwood 2005, Wachter et al. 2014). Prominent — mutually not exclusive — evolutionary theories of senescence, such as William's antagonistic pleiotropy hypothesis (Williams 1957), or Medawar's mutation accumulation hypothesis (Medawar 1952), provide general predictions about uniform 
42

senescence patterns across many taxa (Hamilton 1966). However, these generalities have been questioned both theoretically and empirically by illustrating how negligible and negative-senescence can theoretically be achieved and been empirically found in various species (Vaupel et al. 2004, Jones et al. 2014). Despite an extensive literature on mechanistic approaches of aging, the generating processes that drive such diversity in senescence patterns remain opaque (López-Otín et al. 2013). Mechanistic approaches to aging identify a multitude of underlying biochemical, molecular and organismal mechanisms that relate to the decline of function with age, many of which are rooted in direct or indirect oxidative processes (Kirkwood 2005, López-Otín et al. 2013). Examples include age-related mitochondrial dysfunction, telomere shortening, stem cell exhaustion, genotypic instability, epigenetic alterations, accumulation of damaged proteins and general loss of proteostasis (Lindner and Demarez 2009, Tyedmers et al. 2010, López-Otín et al. 2013). Yet, researchers have not conclusively determined whether such mechanisms are a cause or consequence of aging. This failure may be due to the complexity of model systems of aging. As a consequence, it is difficult to relate these mechanisms directly to the observed demographic patterns (Tyedmers et al. 2010, López-Otín et al. 2013, Denoth Lippuner et al. 2014). Only such linkage — between mechanisms and senescence patterns — can elucidate generating processes that underlie the various theories and aspects of aging.

Aging in bacteria has been established over the last one and a half decades and thereby provided a simple biological system to study aging (Ackermann et al. 2003, Stewart et al. 2005). Before, bacteria have been thought to not age (Williams 1957), because they normally fission into two equal sized progeny. These progeny were assumed to be identical, that is, the original mother cell would die when fissioning and leaves two identical daughters (Johnson and Mangel 2006, Tyedmers et al. 2010). This perspective has changed because the resulting progeny are phenotypically unequal (Tyedmers et al. 
2010). This asymmetry among the progeny is manifested by asymmetry of intracellular content at cell fission and between carrying old and newly formed cellular poles (Stewart et al. 2005, Lindner et al. 2008). Here, we follow the convention of old pole and new pole cells, being referred to as mother and daughter cell respectively (Fig. 1), to track the age of individual cells (Stewart et al. 2005). It has been shown that mother cells have a higher probability to accumulate misfolded protein and grow slower as compared to their daughter cells, but a causal relationship to mortality was not established (Lindner et al. 2008). From a theoretical point of view asymmetry is required to rejuvenate some cells in order to prevent whole population aging. Otherwisepopulations would accumulate more and more damage if perfect symmetric fissions occurred and damage accumulation accedes damage repair and dilution due to growth and fission (Ackermann et al. 2007, Evans and Steinsaltz 2007).

Here, we reveal characteristics of the underlying aging processes by inference from observed senescence patterns. We achieve this aim by quantifying demographic parameters of a simple biological system, isogenic individual E. coli bacteria cells, under highly controlled environmental conditions. We used a high-throughput microfluidic device (Fig. 1; movie S1) to track individual cells throughout their lives (Wang et al. 2010, Gasset-Rosa et al. 2014, Jouvet et al. 2017). Two types of cells were tracked: early daughter cells and late daughter cells (Fig.1). A late daughter cell is the last daughter cell produced by an early daughter and hence an offspring of an old mother. An early daughter cell is the offspring of a young mother since they were haphazardly extracted from a population that grew exponentially. According to stable stage population theories such exponentially growing populations are vastly dominated by young cells (Caswell 2001)(Fig. S1). We expect early daughters to hold little or no deterioration or damage at birth, whereas late daughters are likely be born with some damage. In this study, we use damage as a synonym for any unknown aging factor that leads to deterioration and 
increased mortality. We use this synonym because many aging factors are assumed to be accumulated damage caused by oxidative processes (Kirkwood et al. 2005, López-Otín et al. 2013). A third group of cells, resembling the late daughter type are the last daughter cells produced by the late daughters, which we call second generation late daughters (Results only in SI1). Our definition of mother and daughter cells builds on the concept of cell polarity for both early daughter and late daughter cells to track the age of cells and distinguish between the mothers (old pole cell) and the daughters (new pole cell) (Fig.1).

\section{Material and Methods}

For brevity we only provide in this section an overview of the methods. For more detailed methodological information on strains and growth conditions, time-lapse imaging, image analysis, determining death and estimating demographic parameters, statistical analyses, the simulations please see the supplemental information.

We collected data in two independent sets of experiments. We loaded E. coli K12-derived MG1655 strain cells into a designed microfluidic (PDMS) chip (Wang et al. 2010, Gasset-Rosa et al. 2014, Jouvet et al. 2017) from an exponentially growing culture in supplemented minimum media M9. During each experiment, we acquired 77 hours of time lapse phase-contrast imaging (15 frames/hour for each of the $2 \times 44$ fields followed) using a temperature-controlled inverted microscope, at $43^{\circ} \mathrm{C}$ with an accuracy of the temperature control at the chip of $\pm 0.1^{\circ} \mathrm{C}$ (note within the chip temperature should be even more closely controlled due to some buffering of the chip). We used $43^{\circ} \mathrm{C}$ to accelerate the aging process and thereby shorten the time the system needed to run under stable conditions. Such stability was particularly important to accurately track late daughter and second generation late daughter cells. Increasing temperature up to $43{ }^{\circ} \mathrm{C}$ scales senescence patterns, but does not alter the shape of patterns (Jouvet \& 
111 Steiner unpublished). At $44{ }^{\circ} \mathrm{C}$ patterns got unstable and cells were not viable over longer time periods 112 (results not presented). The rod-shaped bacteria cells grew in dead end side channels of a microfluidic

113 chip, with the focal cells trapped at the end of the side channels, and we tracked them over their lifespan 114 (Fig. 1; movie S1). We used customized image analysis to generate the demographic data (lifespan, cell 115 elongation rate, cell size, and time of each division).

117 We assured by starting the experiments with exponentially growing cells that the initial loaded cells are 118 descendants of young mothers (i.e. they are early daughter cells) (Fig. S1). At the end of their lives, these early daughter cells produced a last daughter (late daughters) that then became the next bottom-most cell trapped at the end of the respective growth channel. Therefore, we could directly compare mother and daughter cells. Note that the late daughters are not born at the same time (main text Fig. 2 E, F). The late daughter cells produced another generation of late daughter cells at the end of their lives (second generation late daughter cells) for which the results are shown in Fig. S2-8, Table S1-3. Analysis on the empirical data were done in program R (R Core Team 2016) using general linear, generalized linear, and non-linear models. Models were selected based on information criteria (AIC) (Burnham and Anderson 1998) or based on differential evolution algorithm for global optimization (R package DEoptim).

Simulations and extending random deterioration models.

For the extended random deterioration model, we first estimated parameters by fitting a fixed frailty model - a Gamma-Gompertz-Makeham (GGM) model — to the observed survival data of the early daughter cells (Yashin et al. 1994, Missov and Vaupel 2015). We then translated these fitted GGM model parameters to an extended random deterioration process model, an extended LeBras type deterioration model (Le Bras 1976). In doing so, we took advantage of mathematical similarities between the two 
134 types of models, even though they are biologically distinct (Yashin et al. 1994, Missov and Vaupel 2015).

135 With these random deterioration model parameters we could estimate the probability matrix of an 136 individual being at stage $i$ at age $x$. Microsimulations based on these models provided stage at death 137 distribution. For the late daughters, we assumed that they are born at a scaled version of the same damage 138 stage in which their mothers died. We also assumed that late daughters accumulate damage at the same 139 rates as early daughters do, i.e. same probabilities to transition to a higher damage state of early and late 140 daughters. We further assumed that the amount of accumulated damage in late daughters had the same 141 effect on mortality than on early daughters, that is, early and late daughter cells are not fundamentally 142 different except that late daughters are born in different damage stages, while early daughters start their 143 lives without damage, but the baseline mortality (Makeham term) can be interpreted as some starting 144 level of damage even for early daughters. Our model simplifies the biological system substantially in as 145 much as no repair of damage or purging of damage through asymmetric division at cell fission is 146 considered. Damage accumulates unidirectionally, mortality increases exponentially with accumulated 147 damage, and each cell suffers from an age-independent baseline mortality risk (Makeham term). 


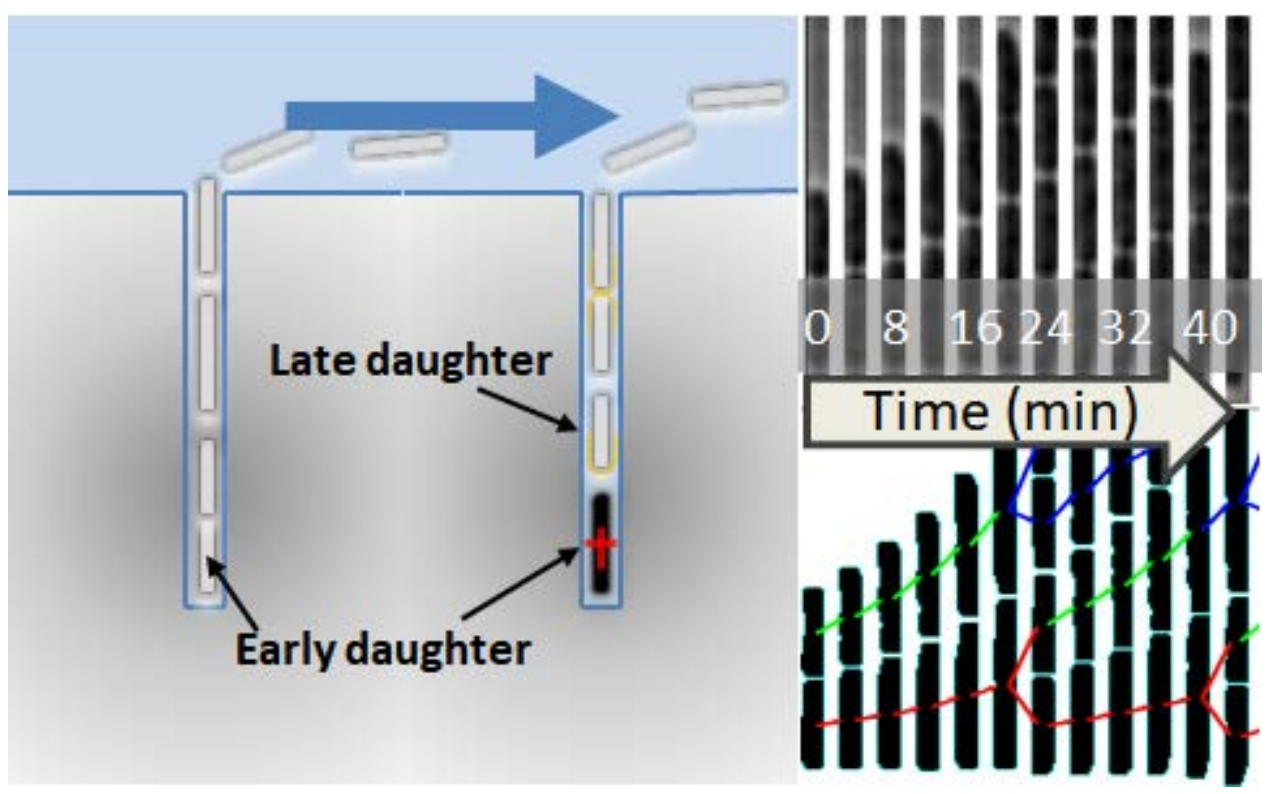

151 Fig. 1: Left panel: Overview sketch of the microfluidic device with dead-end side channels and the main 152 laminar flow channel where the media is flushed through. The early daughters (founding initial loaded mothers) are the bottommost cells of the dead-end channels. Their daughters (new pole progenitor cells) are located closer to the main laminar flow channel and have more recent poles. When the mother (early daughter) dies (side channel on the right), its last daughter ( $1^{\text {st }}$ generation late daughter $)$ is then tracked throughout its life. Accordingly, $2^{\text {nd }}$ generation late daughter cells are tracked once their mother $\left(1^{\text {st }}\right.$ generation late daughter) dies. Right top and bottom panel: Phase contrast time sliced (4 min intervals) side channel images with initial loaded early daughter at the bottom (old pole progenitor). Growth (cell elongation) and division can be tracked throughout their life as depicted in the segmented cell lineages (bottom right panel). See also movie S1.

\section{Results and Discussion}


164 In our experiments, we followed by automated time-lapse microscopy 516 early daughters throughout 165 their lives as they divide and age in microfluidic dead-end channels (Fig. 1). We also followed two 166 generations of late daughters of these 516 early daughters and recorded each cells growth rate and 167 lifespan. In this, we reveal classical senescence patterns of a decrease in reproduction (Fig. 2A, B) 168 indicated by decreased cell elongation and increased size at division with age (Fig. S2, Table S1) — for 169 both early and late daughters (Figure 2A, B). The observed senescence patterns (Fig. 2C, D) describe 170 chronological aging in E. coli and support previous studies on replicative senescence (time counted as 171 number of divisions) in this species (Lindner et al. 2008, Wang et al. 2010) (Fig. S2 \& Fig. S4). The 172 main result highlights that early daughters and late daughters differ fundamentally in their senescence 173 pattern, even though they are isogenic and grown in a highly controlled environment. Only the early 174 daughters exhibit the classical senescence pattern, marked by an early exponential increase of the 175 probability of death followed by a later age mortality plateau. Late daughters have the same probability 176 of death across most of their lives, i.e. no senescence is observed at the population level. Only late in life 177 does mortality increase, but this increase is largely driven by only one data point, the one for the last age 178 class $(>30 \mathrm{~h})$ and is accompanied by increased uncertainty due to small sample size at that age. Such a 179 plateau, exhibited by the early daughters — recurrent in many higher organisms including humans 180 (Vaupel et al. 1998) — has not been previously shown for bacteria, potentially indicating deep-rooted 181 features of aging and senescence. In yeast, ambiguous results on senescence patterns have been described 182 (Minois et al. 2005, Denoth Lippuner et al. 2014). 

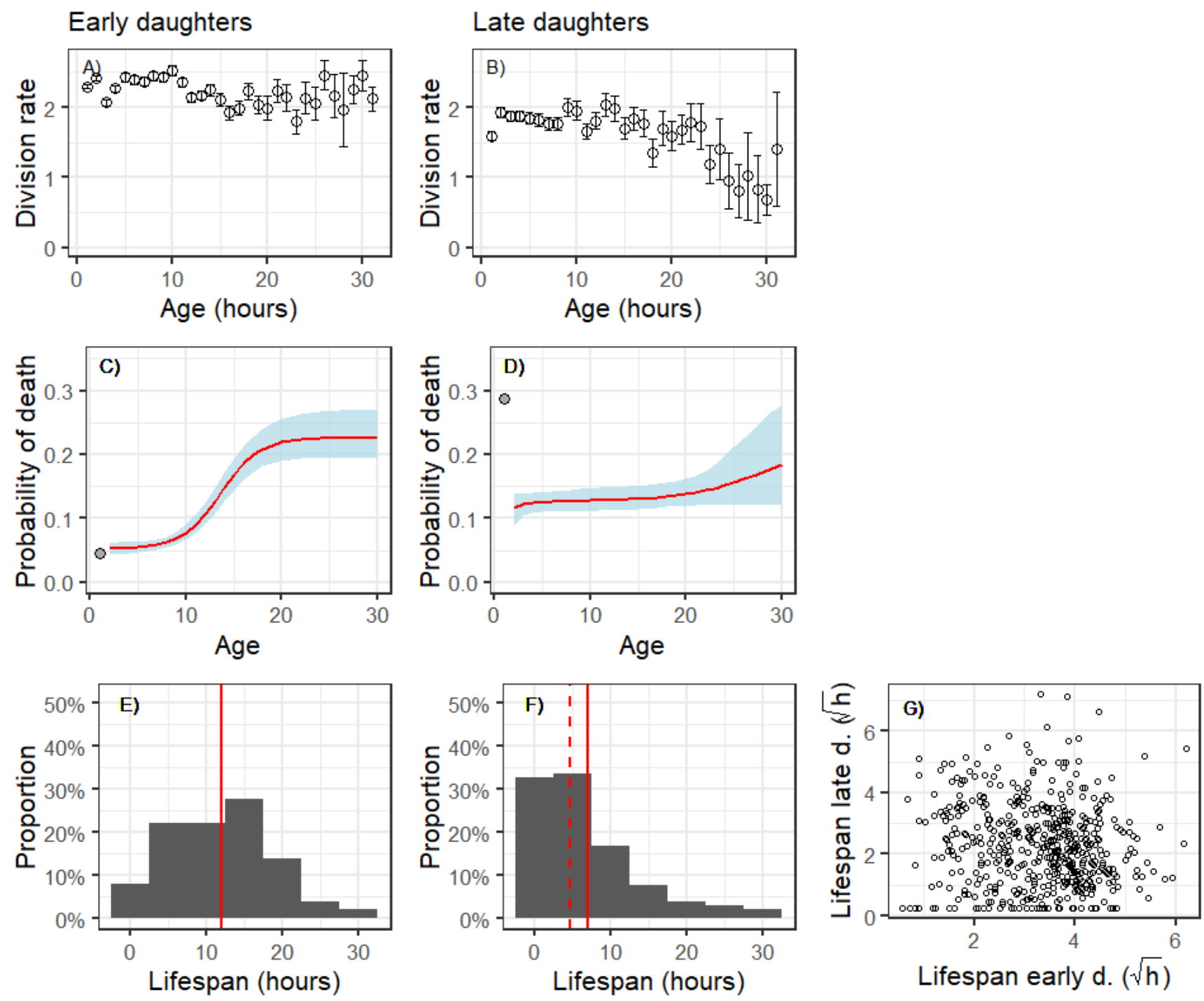

184 Fig. 2: Division rate (divisions per hour) (A, B) and probability of death per hour (C, D) with increasing 185 age, as well as lifespan distribution (in hours) (E, F) of isogenic E. coli cells grown under highly controlled environmental conditions in a microfluidic device (Fig. 1, movie S1). Age patterns are shown 187 for early daughters (A, C, E; N=516), and late daughters (B, D, F; N=516). Late daughters are the last 188 offspring of the founding early daughters. The correlation of early daughter's lifespan (mothers’ lifespan) 189 (square root transformed for better visibility; $\mathrm{N}=516$ ) versus the lifespan of their last daughter cell (late 190 daughter cells; $N=516)$ is shown in panel $(G)$. For $(A, B)$ hourly means \pm standard errors are plotted, for 
191 (C, D) the fitted regression $\pm 95 \%$ confidence intervals are plotted (confidence intervals are estimated 192 based on individual level data SI1). The fitted regression (red lines) are likelihood optimization for 193 Gamma-Gompertz-Makeham functions and relate closely to the modeling approach we took below (C, 194 D, see also Fig. 3, simulations below, and SI1; Table S1, Table S2 for statistical testing) (Burnham and 195 Anderson 1998). The grey points in panel C, D, mark average first hour probability of death which have 196 been excluded from the modeling. For lifespan distributions (E, F), mean and median are marked by solid 197 and dashed lines respectively. For patterns of the second generation late daughters, see Table S2, Table 198 S3, Table S4, and Fig. S3, Fig. S7, Fig. S8, Fig. S9, Fig. S10, Fig. S11.

The probability of death of newborn late daughters (<=1hour, filled grey data point in Fig. 2D) drops 201 after the first hour to a level that is lower than their long-lived mothers (early daughters above age 20) and then remains at that level throughout their lives. This drop in mortality early in life of late daughters suggests a damage purging effect (see below). Such a drop of mortality after birth might indicate 204 heterogeneity among newborns amenable to evolutionary selective forces (Yashin et al. 1994), e.g. 205 heterogeneity in maternally-transmitted damage between mothers and daughters. With increasing age we 206 detected increased variance and increased uncertainty of parameter estimation (Fig. 2 A-E). Such increase is expected for age-structured demographic analyses and well understood for its declining number of individuals with age (Brillinger 1986, Klein and Moeschberger 2003, Scherbov and Ediev 209 2011). The increase in variance is more pronounced for probabilities of death (Fig. 2 C, D) than for division rates (Fig. 2 A, B), because each individual dies only once while each individual divides multiple times throughout its life and hence division rates suffer less from smaller sample sizes. Binning over larger time spans or having an earlier open age bracket would diminish the effect of increased variance 213 with age. 
Damage accumulation and selection

216 If we assume, as in most physiological theories of aging, that accumulated damage is the determining 217 factor for the probability of death, then the observed constant probability of death with age for older early 218 daughters and the late daughters (Fig. 2C, D) indicates an equilibrium distribution of damage among 219 individuals. Such equilibrium is realized at the population level while the individuals accumulate damage in a stochastic manner. It equilibrates on one hand the accumulation of new damage within individuals similar to processes described by random deterioration models (Weitz and Fraser 2001), and on the other hand, the intracellular repair of damage and purging of damage by two mechanisms. The first mechanism reduces damage within an individual by asymmetric division of damage at cell fission, which increases variance in damage among individuals (mother and daughters). The second mechanism selects against damage in the surviving population, and reduces the variance of damage among individuals (Evans and

Table S3) than the late-age mortality plateau of the early daughters (Fig. 2C). This indicates — based on

fixed frailty models (Missov and Vaupel 2015) — higher heterogeneity in transmitted damage of late 
236 reminiscent of asymmetric partition of protein aggregates in bacterial cells due to their passive 237 accumulation in bacterial mother cells (Lindner et al. 2008, Coquel et al. 2013).

239 Stochastic variability in lifespan and lack of mother-daughter correlation of lifespan

240 We did not find any influence of the lifespan of the mothers on the lifespan of their (last) daughters (Fig.

$2412 \mathrm{G}$ ) (Table S6, Fig. S8). Since we are working with an isoclonal population we do not expect any genetic 242 variability among mother-daughter pairs; therefore, we would not expect a genetically driven mother243 daughter correlation of lifespan caused by additive genetic differences. However, non-genetic factors 244 such as maternal transmitted damage would predict mother-daughter correlated lifespans except if i) 245 daughters would be perfectly rejuvenated — all daughters would be born without damage (see also Fig. $2463 \mathrm{E})$ - , or ii) if the transmission of damage to the daughter is independent of the amount of accumulated 247 damage by the mother — the mother-daughter transmission of damage is stochastic. Since late daughters 248 display higher probability of death from birth onwards (Fig. 2D), which is indicative of some maternally transmitted damage and obviously shows that late daughters are not perfectly rejuvenated, we can exclude the first explanation why a mother-daughter correlation of lifespan is lacking. This leaves the second explanation where the amount of damage transmitted from the mother to the daughter is stochastic and independent of the age of the mother. Early daughters seem to hold little or no damage at birth, since their mothers have not yet accumulated much damage, whereas the average late daughter seems to be born with some damage that raises its probability of death. The lack of a mother-daughter correlation of 
258 The lack of correlation between mother-daughter lifespan suggests that the assumed mother-daughter 259 transmission of damage is predominantly stochastic. If damage accumulates stochastically within a cell 260 over its life, long-lived mothers might have been lucky and accumulated damage at a lower rate than the 261 average mother, but they still accumulate damage with increasing age. At age of death, long-lived 262 mothers might have similar damage levels compared to short lived mothers. Short-lived mothers, which 263 likely accumulated damage at an exceptional high rate, do not produce daughters with shorter life expectancy. Despite their difference in lifespan, neither long-lived nor short-lived mothers tend to transmit higher or lower amounts of damage to their daughters, otherwise we would find a negative or positive correlation in mother-daughter lifespan (Fig. 2G). Therefore, our observation that early daughters — which are assumed to hold little or no damage at birth — die at very different ages indicates that the process of damage accumulation within an individual appears to be stochastic and varies highly among individuals. Despite an isogenic population in a highly controlled homogeneous environment, we observe a high variation in lifespan and reproduction among individuals, both for early daughters 271 (Median \pm stdev lifespan $12 \pm 7$ hours, Coefficient of Variation, CV 0.57, Fig. 2E) (Fig. S7) and late daughters (Median \pm stdev $5 \pm 7$; CV=1.01, Fig. 2F). Such high variation supports substantial influences of stochastic events in shaping lifespan.

Modeling damage accumulation and asymmetric transmission of damage

To support our conclusions based on the empirical results and to gain a better understanding of the underlying stochastic processes that shape our observed senescence patterns, we extended random deterioration process models (e.g. random accumulation of damage, see details in Methods and SI1) (Le Bras 1976, Yashin et al. 1994, Evans and Steinsaltz 2007). Our extended model assumes that all cells accumulate damage unidirectionally at the same rate, i.e. cells move with equal probability to a higher 
281 damage state, mortality increases exponentially with accumulated damage, and each cell suffers from an age-independent baseline mortality risk. We also assume that early daughters are born without damage

(Fig. 3A). The results of our simulations show that with increasing age population level damage (and variance) slowly increases at early ages, accelerates after 10 hours and plateaus after 20 hours (Fig. 3A).

These patterns in damage accumulation mirror the observed mortality patterns (Fig. 2C). Mortality plateaus, as observed for early daughters, can be explained by either fixed frailty, i.e. heterogeneity in

birth seems to have little influence, at least for early daughter cells. Senescence is driven by an age independent baseline mortality and accrued damage as in random deterioration models (Le Bras 1976,

Yashin et al. 1994, Weitz and Fraser 2001). This conclusion is supported by two results of our model.

First, most early daughters die with no or very little damage (Fig. S10), and second, the observed survival patterns follow closely the survival curve of simulated cells that did not accumulate any damage throughout life (Fig. 3C).

Contrasting the assumption for the early daughter cells, in our simulation the average simulated late daughter is born with some damage ( 24\% are born without damage) (Yashin et al. 1994)(Fig. 3B). For the following results, it is important to note that this heterogeneity in damage at birth of late daughters is an underestimation since we do not include the exceptionally high first-hour mortality rate (grey filled data point in Fig. 2D) for the simulations. In our simulations the distribution of this damage at birth of 301 the late daughters is related to the distribution of the accumulated damage at death of the early daughter cells (mothers of the late daughters, Fig. S10). For the simulations, we assumed that the mother to daughter transmission of damage is a fixed fraction of all the damage the mother accumulated over her 
lifespan. Note, we only simulated a single binomial division, rather than multiple asymmetric fissions as

305

306

307

308

309

310

311

312

313

314

315

316

317

318

319

320

321

322

323

324

325

326

others have done (Evans and Steinsaltz 2007). We computed the fixed fraction of damage transmission between mother and daughter by optimizing the survival pattern predicted by the simulations to the observed survival curves.

We found that the optimal (simulated) fixed fraction was at a low level of 0.07 . This indicates a highly asymmetric division of damage between mothers and daughters. If damage transmission between mothers and daughters would be symmetrical (0.5), the predicted survival of the simulations (Fig. 3D hatched line) does not match the observed survival (Fig. 3D red line). For early daughters, the level of asymmetry makes little difference because most of them die with no or little accumulated damage (Fig. 3C, Fig. S10). The predicted survival pattern of late daughters at older ages slowly converges to the simulated survival patterns of undamaged cells (outer right grey line) (Fig. 3D). Therefore, many cells in our simulation that survive to old ages hold little damage, which supports our interpretation of our experimental data that late daughter cells are born with diverse damage states, highly damaged cells are selected against, and at old ages, population-level survival patterns are strongly influenced by cells that have not accumulated much damage.

Similar selective effects have been described by population level demographic theories as heterogeneity’s ruses, where selection among different damage stages (fixed frailty; i.e. heterogeneity in damage stage at birth) leads to diverse senescence patterns at the population level (Vaupel and Yashin 1985). Compared to our simple model that only considers a single binary fission (branching) and suggests a strong asymmetry, more complex and realistic models that include multiple branching (fission) events show less extreme asymmetry at cell fission to be adaptive (Evans and Steinsaltz 2007). This difference 
327 in optimal asymmetry between our simple and the more complex models can be understood in that the 328 asymmetric transmission of damage is distributed over multiple fissions in the more complex models, 329 while for our model it is concentrated at a single event. Even though the optimized fixed transmission 330 fraction between mother and daughters of our model is low at 0.07 , such a fixed fraction would lead to a 331 correlation in lifespan between mother and daughters of about 0.25 (Fig. 3E). If different levels of fixed 332 transmissions are simulated, correlation in lifespan between mother and daughters increases with 333 increasing transmission. Only when there is no transmission of any damage (perfect rejuvenation of the 334 daughters) we see no correlation in lifespan (Fig. 3E). The observed data does not support such 335 correlation in lifespan (Fig. 2G), but also does not support perfect rejuvenation (Fig. 2C and D). Hence 336 these simulations support our conclusion that the fraction of transmitted accumulated damage from 337 mother to daughter at cell fission varies stochastically and is not fixed at a low level of 0.07 as our model assumes. 
Early daughters
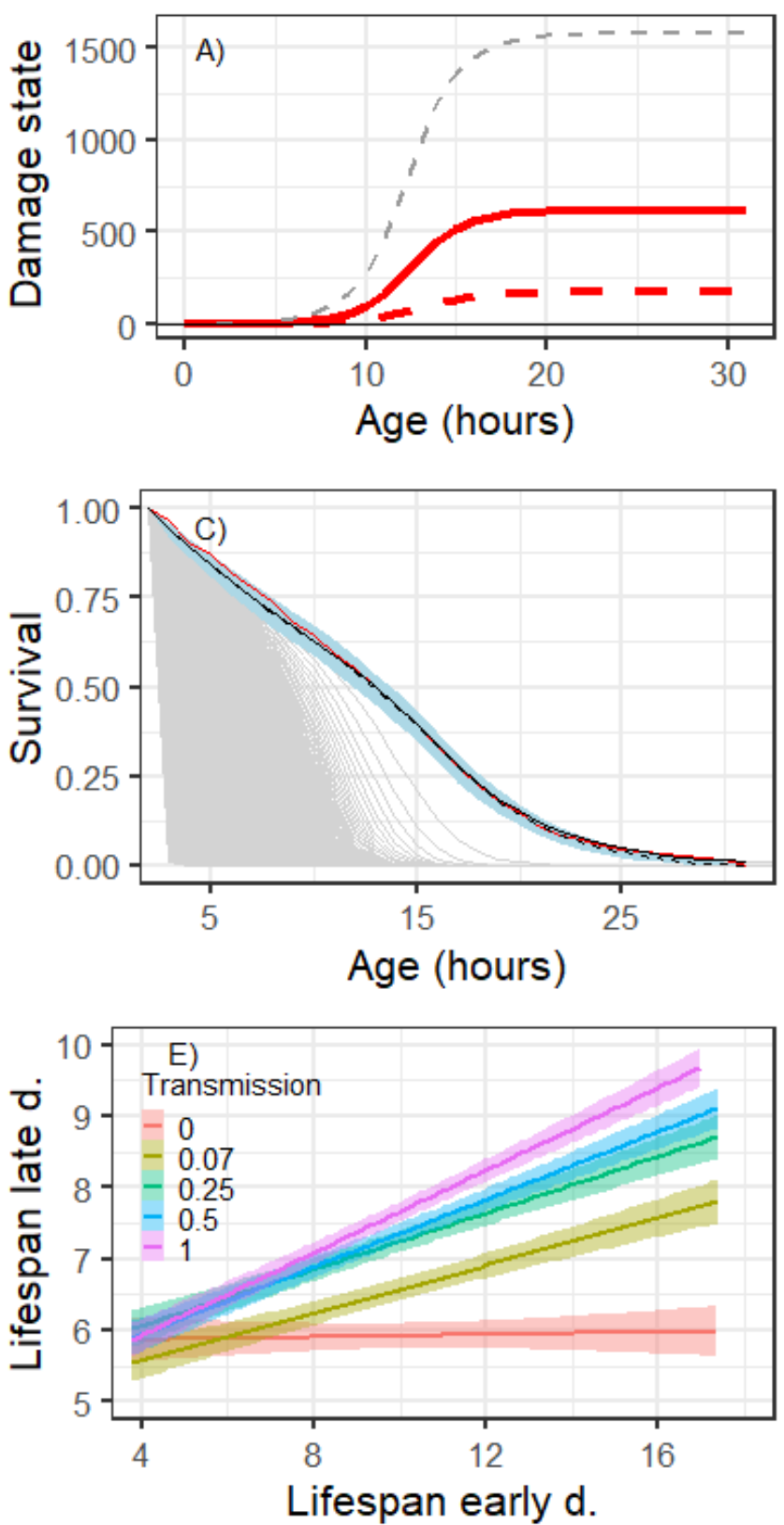

Late daughters
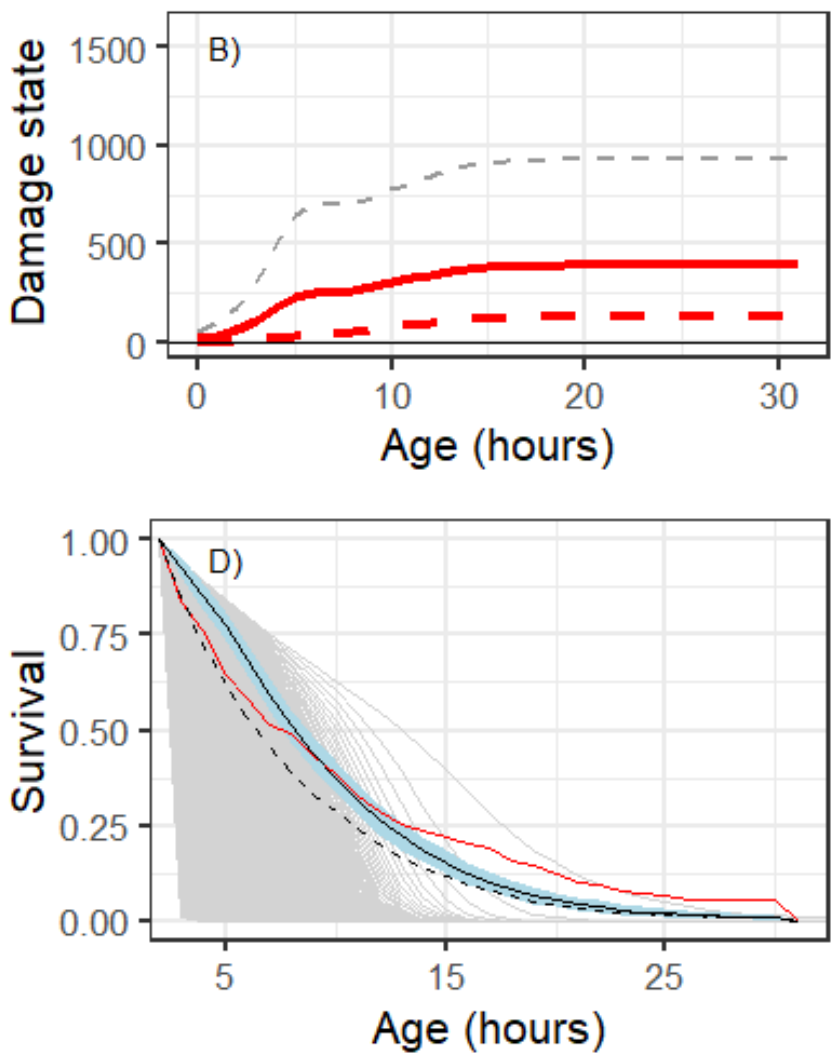

Fig. 3: Random deterioration model: Simulation results are shown of mean (red solid line, + SD grey

343 hatched lines) and median (red hatched line) damage state with increasing age (A, B); observed experimental population level survival curves (red solid line; see also related probability of death Fig. 2 
345 C, D and related observed distributions of death Fig. 2E, F), Gamma-Gompertz-Makeham simulated 346 survival curves with symmetric damage transmission (hatched black line) as well as simulated 347 asymmetric damage transmission of $7 \%$ (solid black lines $\pm 95 \%$ CI in blue) (C, D). Graphs are shown 348 for early daughter cells (A, C) and late daughter cells (B, D). Thin grey lines in (C, D) depict expected 349 survival curve of simulated cells with different fixed damage state: outermost (right) curve depicts the 350 highest survival of simulated cells with no damage throughout their lives, most left survival curve depicts 351 the lowest survival of simulated cells that were born with maximum damage level of 5000. Lifespan 352 (square root transformed) of simulated 516 early daughter cells (mothers) versus the lifespan of their 353 simulated last daughter cell (late daughter cells) with different levels of mother to daughter damage 354 transmission (E). The different scenarios include the optimized fixed transmission level at 0.07 (forest 355 green), a scenario for perfect rejuventation, i.e. 0 transmission (red), 0.25 transmission (green), 0.5 356 transmission, i.e. symmetric (equal) transmission between mother and daughter (blue), and transmission 357 of all accumulated damage to the daughter (1) (pink).CI are shown for each correlation in lifespan 358 between mothers and daughters as shaded areas.

In this study, our experimental setup limits our analysis to two extreme cases: early and late daughter cells (in fact the last daughter cells). We hypothesize that the results do not only hold for these two extremes but rather portray the extremes of a continuous process across different aged cells. Two observations support our claim: first, changes in mortality of early daughters (Fig. 2C) and age patterns 364 in reproduction (Fig. 2A) are somewhat gradual (more so for survival than for reproduction), suggesting 365 a gradual underlying mechanism; second, we do not observe a pronounced pattern just before death (Fig. 
367 their mothers are approaching death, for instance in the way as predicted under terminal investment theories (Clutton-Brock 1984).

$\underline{\text { Two stochastic processes shape diverse senescence patterns }}$

371 We conclude that the diverse senescence patterns, including the classical senescence pattern of a 372 mortality plateau, are determined by two stochastic processes that relate to underlying (damage) states 373 and only indirectly to age (Yashin et al. 1994, Wachter 1999, Weitz and Fraser 2001, Evans and Steinsaltz 374 2007). The primary process is a random deterioration process, e.g. the stochastic accumulation of damage 375 throughout life, and the secondary process involves the stochastic transmission of damage from the 376 mother to their daughters at cell fission. This transmitted damage or some other unknown aging factor increases the probability of death but is "non-heritable” as we show by the lack of correlation between mother and daughter lifespan (Yashin et al. 1994, Wachter 1999, Weitz and Fraser 2001, Evans and 379 Steinsaltz 2007). Not only additive genetic variation but also other commonly assumed drivers of senescence, such as epigenetic variability, age itself, or the (extrinsic) environment can be ruled out as major players in our study. If epigenetic variability had a significant influence, a positive correlation among mother and daughter lifespan would be seen. If chronological age determined senescence, early and late daughter cells would show similar mortality patterns, hence senescence is rather driven by stage dynamics than by chronological age. The highly controlled microfluidic system creates a uniform environment and hence we can exclude extrinsic environmental drivers; this does not imply that senescence patterns do not differ under different environmental conditions, we just investigated only one specific environment. For instance, under complex media variance in division size and related division rate is increased compared to the minimum medium we used (Gangan and Athale 2017). Minimum medium also decreases the rate of filamentation, a stress response that causes continued cell elongate 
without dividing. Recovery of filamentous cells is rarely observed under minimum medium but frequent

under complex medium (Wang et al. 2010) (see also SI). The near identical conditions cells experience

in the microfluidic system decreases environmental variability compared to naturally experienced micro-

habitats. The laminar flow provides large amounts of fresh media that constantly diffuses within seconds

into the dead-end side channels and therefore prohibits built-up of micro-environmental-niches. Though,

such micro-environments (e.g. biofilms) are characteristic for the natural conditions in the guts of the

mammalian hosts most E. coli life and have evolved under. The microfluidic system we use largely

prevents cell-to-cell contact and chemical communication among cells that can influence population

growth (Aoki et al. 2005). The highly controlled conditions therefore lowers phenotypic variability

compared to natural conditions. To what degree senescence patterns are altered by the environment

remains to be explored. Temperature seem to conserve the shape of senescence patterns and simply scales

patterns differently, whereas nutrient concentrations and nutrient source might alter shapes as has been

found in other organisms (Stroustrup et al. 2016)(Steiner unpublished).

403

404

Our findings indicate that two simple stochastic processes can create complex senescence patterns. Easily

understood, simplistic, and plausible arguments behind evolutionary theories of aging might lure readers

into the misconception that chronological age in itself plays an important role in senescence. Such

simplistic assumptions of age-specific drivers must be approached with caution and seem not applicable

to this study. Selective forces likely decline with time in our system, but the decline might be dominated

by underlying stage dynamics. Selection on heterogeneity among individuals is rather generated by such 
413 events on life histories is also indicated by the large variability in life histories despite excluding any

414 genetic or environmental variability. Such large stochastic variation supports arguments behind neutral

415 theories of life history (Steiner and Tuljapurkar 2012) that suggest that stochastic variability can account

416 for substantial fractions of the total variability in fitness components. Our interpretation of stochastic

417 events determining individual life courses is consistent with findings of significant stochastic influences

418 at the molecular (Elowitz et al. 2002) and protein level (Tyedmers et al. 2010, Balázsi et al. 2011) and

419 adds to the growing interest in such phenotypic heterogeneity and individuality (Wolf et al. 2007,

420 Davidson and Surette 2008, Ackermann 2015).

421

422 Future challenges will include determining whether these stochastic processes are neutral or adaptive, 423 and what drives their evolution (Steinsaltz and Evans 2004, Kærn et al. 2005, Norman et al. 2015, Vera 424 et al. 2016). For the basic aging processes that drive senescence, the causal relationships that drive age 425 patterns are currently unknown (Lindner and Demarez 2009, López-Otín et al. 2013). The complex senescence patterns found in this study of a simple model organism under highly controlled conditions emphasizes the challenges to quantify contributions of well-defined determinants of aging in the complex systems on which most aging research is focused (López-Otín et al. 2013). Comparing mean characteristics of differently aged individuals as frequently done in aging research might hold limited insights in system where determining stages are likely highly dynamic. In light of the growing evidence that stochastic processes can have cascading effects across all levels of higher organisms (Finch and 432 Kirkwood 2000, Elowitz et al. 2002, Balázsi et al. 2011) new avenues in aging research may require a 433 shift towards the underlying stochastic processes that drive such stage dynamics in simple systems like 434 bacteria and perhaps beyond. Identifying the underlying currently unknown (damage) states remains 435 another challenge that we believe requires a combined quantitative demographic and mechanistic 
approach, because of the high level of stochastic influences. Promising steps in such directions have been

initiated by theoretically exploring stage-specific alleles shaping senescence patterns (Wachter et al.

2014) and by exploring transcription factor signal dynamics at the single cell level across increasing parts

of the lifespans of many individuals (Norman et al. 2013).

\section{Acknowledgements}

444 We thank D.A. Roach, D. Steinsaltz, T. Coulson, Y. Yang and S. Tuljapurkar for discussions and

comments.

\section{References}

Ackermann, M. 2015. A functional perspective on phenotypic heterogeneity in microorganisms. - Nat. Rev. Microbiol. 13: 497-508.

Ackermann, M. et al. 2003. Senescence in a bacterium with asymmetric division. - Science 300: 1920.

Aoki, S. K. et al. 2005. Contact-Dependent Inhibition of Growth in Escherichia coli. - Science (80-. ). in press.

Balázsi, G. et al. 2011. Cellular decision making and biological noise: from microbes to mammals. Cell 144: 910-925.

Blattner, F. R. 1997. The Complete Genome Sequence of Escherichia coli K-12. - Science (80-. ). 277: 1453-1462.

Brillinger, D. R. 1986. A Biometrics Invited Paper with Discussion: The Natural Variability of Vital Rates and Associated Statistics. - Biometrics 42: 693.

Burnham, K. and Anderson, D. R. 1998. Model selection and multimodel inference : a practical information-theoretic approach. - Springer.

Caswell, H. 2001. Matrix population models: construction, analysis, and interpretation. - Sinauer Associates.

Charlesworth, B. 1994. Evolution in age-structured populations. - Cambridge University Press.

Clutton-Brock, T. 1984. Reproductive effort and terminal investment in iteroparous animals. - Am. Nat. 123: 212-229.

Coquel, A.-S. et al. 2013. Localization of protein aggregation in Escherichia coli is governed by 
diffusion and nucleoid macromolecular crowding effect. - PLoS Comput. Biol. 9: e1003038. Coulson, J. C. and Fairweather, J. A. 2001. Reduced reproductive performance prior to death in the Black-legged Kittiwake: senescence or terminal illness? - J. Avian Biol. 32: 146-152.

Davidson, C. J. and Surette, M. G. 2008. Individuality in Bacteria. - Annu. Rev. Genet. 42: 253-268. Denoth Lippuner, A. et al. 2014. Budding yeast as a model organism to study the effects of age. FEMS Microbiol. Rev. 38: 300-25.

Elowitz, M. B. et al. 2002. Stochastic gene expression in a single cell. - Science (80-. ). 297: 11831186.

Evans, S. N. and Steinsaltz, D. 2007. Damage segregation at fissioning may increase growth rates: a superprocess model. - Theor. Popul. Biol. 71: 473-90.

Finch, C. and Kirkwood, T. B. 2000. Chance, Development, and Aging. - Oxford University Press.

Gangan, M. S. and Athale, C. A. 2017. Threshold effect of growth rate on population variability of Escherichia coli cell lengths. - R. Soc. open Sci. 4: 160417.

Gasset-Rosa, F. et al. 2014. Direct assessment in bacteria of prionoid propagation and phenotype selection by Hsp70 chaperone. - Mol. Microbiol. 91: 1070-87.

Hamilton, W. D. 1966. The moulding of senescence by natural selection. - J. Theor. Biol. 12: 12-45. Jagers, P. 1978. Balanced exponential growth: what does it mean and when is it there? - In: Biomathematics and cell kinetics. Elsevier, pp. 21-29.

Johnson, L. R. and Mangel, M. 2006. Life histories and the evolution of aging in bacteria and other single-celled organisms. - Mech. Ageing Dev. 127: 786-93.

Jones, O. R. et al. 2014. Diversity of ageing across the tree of life. - Nature 505: 169-73.

Jouvet, L. et al. 2017. Demographic variability and heterogeneity among individuals within and among clonal bacteria strains. - Oikos in press.

Kærn, M. et al. 2005. Stochasticity in gene expression: from theories to phenotypes. - Nat. Rev. Genet. 6: 451-464.

Kennedy, B. K. 1994. Daughter cells of Saccharomyces cerevisiae from old mothers display a reduced life span. - J. Cell Biol. 127: 1985-1993.

Kirkwood, T. B. L. 2005. Understanding the odd science of aging. - Cell 120: 437-47.

Kirkwood, T. B. L. et al. 2005. What accounts for the wide variation in life span of genetically identical organisms reared in a constant environment? - Mech. Ageing Dev. 126: 439-443.

Klein, J. P. and Moeschberger, M. L. 2003. Survival analysis : techniques for censored and truncated data. - Springer.

Le Bras, H. 1976. Lois de mortalité et age limite. - Population (Paris). 33: 655-691.

Lindner, A. B. and Demarez, A. 2009. Protein aggregation as a paradigm of aging. - Biochim. Biophys. Acta 1790: 980-96.

Lindner, A. B. et al. 2008. Asymmetric segregation of protein aggregates is associated with cellular aging and rejuvenation. - Proc. Natl. Acad. Sci. U. S. A. 105: 3076-81.

López-Otín, C. et al. 2013. The hallmarks of aging. - Cell 153: 1194-217.

Mattick, K. et al. 2003. Morphological changes to Escherichia coli O157:H7, commensal E. coli and Salmonella spp in response to marginal growth conditions, with special reference to mildly stressing temperatures. - Sci. Prog. 86: 103-113.

Medawar, P. B. 1952. An unsolved problem of biology. - In: Uniqueness of the Individual. H. K. Lewis, in press.

Minois, N. et al. 2005. Advances in measuring lifespan in the yeast Saccharomyces cerevisiae. - Proc. Natl. Acad. Sci. U. S. A. 102: 402-6. 
Missov, T. I. and Vaupel, J. W. 2015. Mortality Implications of Mortality Plateaus. - SIAM Rev. 57: 61-70.

Norman, T. M. et al. 2013. Memory and modularity in cell-fate decision making. - Nature 503: 481486.

Norman, T. M. et al. 2015. Stochastic Switching of Cell Fate in Microbes. - Annu. Rev. Microbiol. 69: 381-403.

R Core Team, R. A. language and environment for statistical computing. 2016. R: A language and environment for statistical computing. (RDC Team, Ed.). - R Found. Stat. Comput. 1: 409.

Scherbov, S. and Ediev, D. 2011. Significance of life table estimates for small populations: Simulationbased study of estimation errors. - Demogr. Res. 24: 527-550.

Steiner, U. K. and Tuljapurkar, S. 2012. Neutral theory for life histories and individual variability in fitness components. - Proc. Natl. Acad. Sci. U. S. A. 109: 4684-9.

Steinsaltz, D. and Evans, S. N. 2004. Markov mortality models: implications of quasistationarity and varying initial distributions. - Theor. Popul. Biol. 65: 319-37.

Stewart, E. J. et al. 2005. Aging and death in an organism that reproduces by morphologically symmetric division. - PLoS Biol. 3: e45.

Stroustrup, N. et al. 2016. The temporal scaling of Caenorhabditis elegans ageing. - Nature 530: 103107.

Tyedmers, J. et al. 2010. Cellular strategies for controlling protein aggregation. - Nat. Rev. Mol. cell Biol. 11: 777-788.

Vaupel, J. W. and Yashin, A. I. 1985. Heterogeneity’s Ruses: Some Surprising Effects of Selection on Population Dynamics. - Am. Stat. 39: 176.

Vaupel, J. W. et al. 1998. Biodemographic Trajectories of Longevity. - Science (80-. ). 280: 855-860.

Vaupel, J. W. et al. 2004. The case for negative senescence. - Theor. Popul. Biol. 65: 339-351.

Vera, M. et al. 2016. Single-Cell and Single-Molecule Analysis of Gene Expression Regulation. Annu. Rev. Genet. 50: 267-291.

Wachter, K. W. 1999. Evolutionary demographic models for mortality plateaus. - Proc. Natl. Acad. Sci. 96: 10544-10547.

Wachter, K. W. et al. 2014. Evolutionary shaping of demographic schedules. - Proc. Natl. Acad. Sci. U. S. A. 111 Suppl: 10846-53.

Wang, P. et al. 2010. Robust growth of Escherichia coli. - Curr. Biol. 20: 1099-103.

Weitz, J. and Fraser, H. 2001. Explaining mortality rate plateaus. - Proc. Natl. Acad. Sci. U. S. A. 98 : 15383-15386.

Williams, G. C. 1957. Pleiotropy, Natural Selection, and the Evolution of Senescence. - Evolution (N. Y). 11: 398.

Williams, G. C. 1966. Adaptation and natural selection : a critique of some current evolutionary thought. - Princeton Univeristy Press.

Wolf, M. et al. 2007. Life-history trade-offs favour the evolution of animal personalities. - Nature 447: 581-584.

Yashin, A. I. et al. 1994. A duality in aging: the equivalence of mortality models based on radically different concepts. - Mech. Ageing Dev. 74: 1-14. 


\section{Supporting information SI1}

556

\section{Strains and growth conditions}

558 We fabricated the microfluidics chips as previously described (Gasset-Rosa et al. 2014). We grew E. coli 559 K12 MG1655 (Blattner 1997) strain derivative (intC::pSulA-yfp) cells at $43^{\circ} \mathrm{C}$ in filtered minimal 560 medium supplemented with $0.4 \%$ Glucose and 0.2\% Casamino Acids (hereafter M9). We diluted 100 $\mu$ l 561 of the overnight culture into $50 \mathrm{ml}$ of $\mathrm{M} 9$ and then grew it at $43^{\circ} \mathrm{C}$ to exponential growth phase (OD600 $562 \sim 0.2$ ). We centrifuged the cells and resuspended the cell pellet in $100 \mu$ l of M9 to load them into the chip 563 by injection into the main (feeding) channel followed by centrifugation (15 minutes at $\sim 168 g$ ). We 564 applied a continuous laminar flow (2.7ml/h) of M9 supplemented with 1.5\% Polyethylene Glycol (PEG 565 P3015 Sigma Aldrich) through the main channel throughout the experiment. The temperature was kept 566 constant at $43^{\circ} \mathrm{C}$ (see below).

567

568

\section{Time-lapse imaging}

569 We followed bacterial growth in the microfluidics chip by phase-contrast time-lapse imaging at a rate of 570 4min/frame using a MetaMorph (Molecular Devices)-controlled inverted Nikon microscope (Eclipse Ti, 571 100xobjective, CoolSNAP HQ2 camera) with a temperature-control chamber (Live Imaging Services). 572 We continuously scanned 44 positions, each comprising 18 channels, for 77 hours, in two independent 573 experimental sets.

\section{Image analysis}

576 We used a custom image analysis to segment all cells within the side channels per frame, the software 577 measured the cells' location and size within the time series and generated cell lineages. To this end, phase 
578 contrast time-lapse images were lowpass-filtered and background flattened (MetaMorph Molecular

579 Devices software) to increase contrast. We further processed the images by a customized ImageJ plugin

580 for cell segmentation to crop each of the growth channels, stabilize the time sequenced images, adjust

581 the brightness and to create a binary image by thresholding and filtering (median filter). Finally, the

582 ImageJ plugin water-sheds the time sliced images. We then applied a final round of segmentation in

583 Matlab by correcting errors in segmentation by checking for minimum (0.8) and maximum (1.4) cell

584 elongation rates for each cell (size time-4 min, size time+4min). This way, we recorded for any time

585 points (at 4 minute intervals) the exact location of a cell in the side channel (the pixel coordinate of the

586 center of mass of the cell area), the side channel of the cell, and if a cell divided. By the position within

587 the side channel we determined which cell was the mother (old pole progenitor) cell (bottommost cell),

588 their most recent daughter (new pole progenitor) cell and so forth. We measured the length of a cell as

589 the largest distance of the rod shaped cell in the orientation of the side channel. We applied a minimum

590 “cell length” of $\sim 1 \mu$ m to exclude artefacts originating mainly from small deformations in the growth

591 channels, or some shadows/reflections generated at the dead end of the growth channel.

592

\section{Age at death}

594 We defined age at death as the time when a cell stopped growing and dividing for at least one hour and $59520 \mathrm{~min}$. None of the cells divided after such a long division arrest or resumed growth.

\section{Early and late daughter cells}

598 We defined the early daughter population as the cells that were initially loaded into the microfluidic chip

599 and settled at the dead-end of the side channels. They originated largely from young mothers because the 600 population was in exponential growth phase when we loaded cells into the chip (Jagers 1978). The age 
601 distribution of these early daughter cells is, because of the exponential growth phase, negatively exponentially distributed. We show their expected age distribution in Fig. S1, based on a Leslie matrix 603 generated from the survival and division rates of the early daughters (Fig. 2A \& C). Due to the 604 exponential growth phase, we assume a stable age distribution (the right eigenvector corresponding to 605 the dominant eigenvalue of the Leslie matrix) (Caswell 2001). We tracked these early daughter cells to 606 the end of their lives (Fig. 1, movie S1). The last daughters that were produced by these early daughter 607 cells are the late daughter population. These late daughters are all of age zero but originate from mother 608 cells (the early daughters) that had accumulated damage over their lives, and that were only one division 609 from dying. We again tracked these late daughters throughout their lives. We focus mainly on these first 610 two types of cells (early and late daughters), though as expected, the next generation of late daughter 611 cells (second generation late daughters) follow the late daughter patterns (Fig. S4-6).

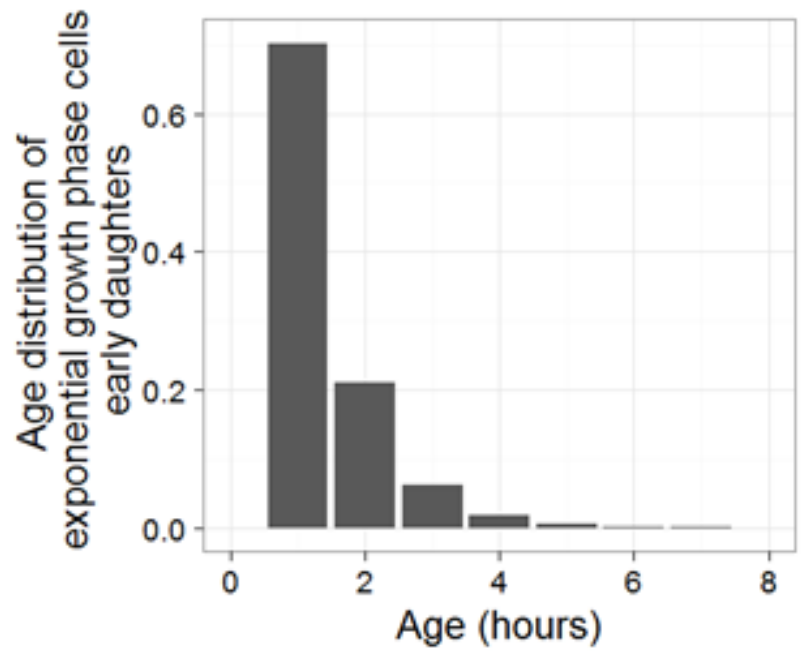

613 Fig. S1: Expected (stable) age distribution (in hours) of early daughters (initial loaded cells), estimated 614 from a Leslie matrix parameterized with the demographic rates of the early daughter cells. Due to the 615 theory of stable age populations, the distribution of the ages of the mothers of these early daughters 
616 should be exactly as the (stable) age distribution shown here of the initial loaded (early daughter cells)

617 at the start of the experiments.

618

\section{Estimating demographic parameters}

620 Image analysis (see above) provided us the basis for estimating the age-specific demographic parameters: 621 division rate, cell elongation rate, size at division, and survival rate. We excluded all cells that were not 622 tracked throughout their lives. This concerned mostly cells that filamented (cell division arrested without 623 growth arrest) and were flushed out of the side channels when they reached a size longer than $25 \mu \mathrm{m}$ 624 (length of the side channel). We also excluded all (early and late daughter) cells that died at chronological 625 age 0 (never started to grow or divide). This was practiced because loading into the chip might be damaging and we wanted to exclude such death. This approach was conservative, because fewer early daughters never started growing or divided compared to late daughters (who could not have been damaged by the loading). We used data from 516 early daughters that produced 516 late daughters which in turns produced 298 (second generation) late daughters that were tracked throughout their lives.

\section{Statistical analysis}

632 We did the statistical analysis using the R software (R Core Team 2016). We estimated and plotted hourly 633 rates for division rates, cell elongation, and cell size at division rather than rates on the 4 min intervals 634 because data would be sparse for 4 min intervals at older ages. Already the hourly rates at old ages suffer 635 from sparse estimates and increased uncertainty. We also clustered all data of ages beyond $30 \mathrm{~h}$ for the 636 same reason. To guide statistical testing, we used model selection (Burnham and Anderson 1998) based 637 on AIC (Akaike Information Criteria) and considered — as common practice — better support between 638 models when the $\triangle \mathrm{AIC}$ was more than 2. For the parameter estimation of the GGM and extended random 
639 deterioration models, we used the DEoptim R package and the optim function within the stats R package.

640 We calculated the CI (Fig. 2 C \& D) by bootstrapping 1000 times. This bootstrapping is based on

641 individual level data not the hourly means Fig. 2 C, D. We choose such an likelihood based global 642 optimization approach to closely relate to the GGM modeling approach we used for the simulations. 643 Using model comparison (AIC) based on the hourly rates (results not shown) selects for models with 644 sigmoidal shapes like the GGM likelihood optimization, i.e. an early exponential increase in mortality 645 followed by a late age mortality plateau (Fig. 2 C). For early daughters, other exponential or linear 646 functions receive less support. For late daughters model comparisons selected non-senescence (Fig. 2 D, 647 flat mortality) over linear or exponential models. We did not test for any breakpoint models or step 648 functions, since such models are not expected based on evolutionary theories of aging.

649

650 Lifespan distribution second generation late daughters

651 In the main article, we focused on early and late daughter cells. Qualitatively, patterns of the second gen 652 eration late daughters (the last daughters produced by the late daughters) correspond to those of their m 653 others (the late daughters), though data becomes sparse uncertainty increases and some deviations occur 654 . Fig. S7 illustrates lifespan distributions of the 298 second generation late daughters, their mothers (late 655 daughters), and grandmothers (early daughters). Kolmogorov-Smirnov tests (two-sided) verifies that bo 656 th lifespan distributions between early and late daughters $(\mathrm{D}=0.3691, \mathrm{p}<0.00001)$, and early to secon 657 d generation late daughters are significantly different $(D=0.4094, p<0.00001)$, while those between la 658 te daughters and second generation late daughters do not differ $(\mathrm{D}=0.0973, \mathrm{p}=0.1189)$. 

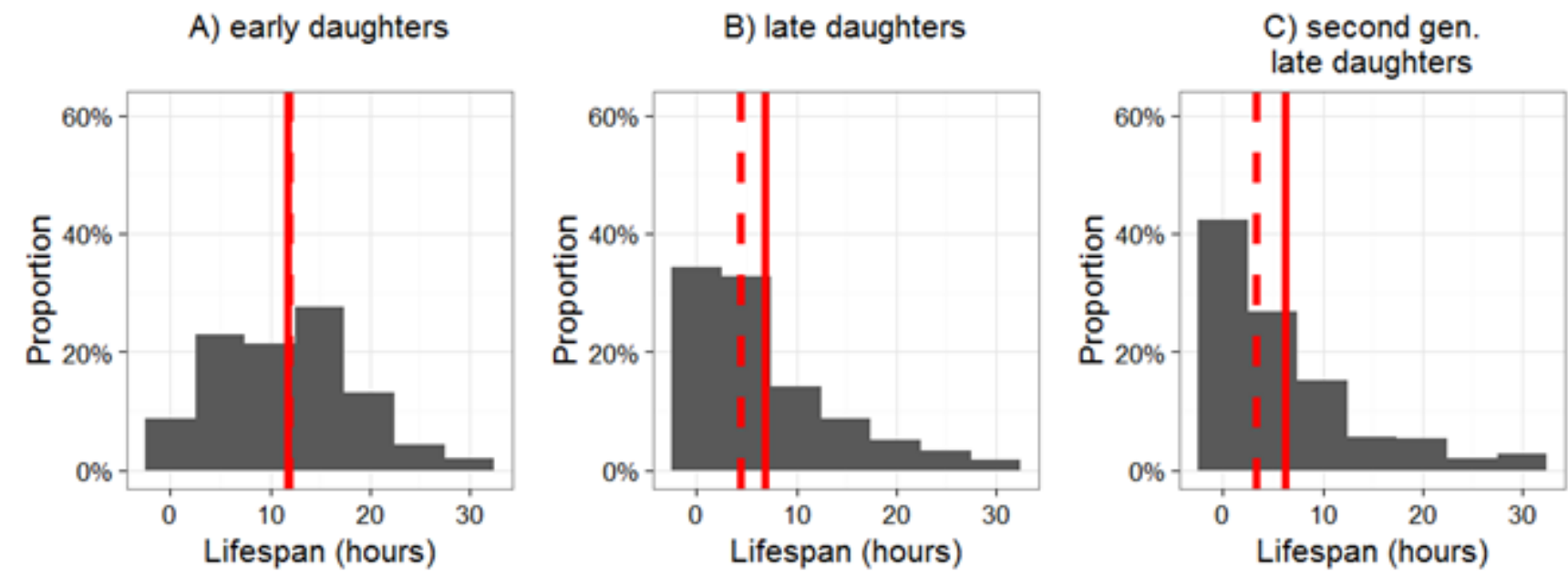

Fig. S7: Lifespan distribution (in hours) of isogenic E. coli cells grown under identical environmental conditions in a microfluidic device (Fig. S1). 1A - Founding early daughter cells (Median \pm stdev lifespan $12 \pm 6.8$ hours, Coefficient of Variation, CV 0.57) ( $\mathrm{N}=298) ; 1 \mathrm{~B}$ - Late daughters (last daughter) of the founding early daughters of 1A (Median \pm stdev lifespan $4.4 \pm 7.0$ hours, Coefficient of Variation, CV

Hence, early daughters are the mothers of late daughters and grandmothers of second generation late daughters.

668

\section{Heritability or cross generation correlation in lifespan}

670 In order to estimate correlation of lifespan between mother cells (early daughters) and their last (late) daughter cells, we used linear models on square root transformed data for age at death (lifespan) for the mothers' lifespan as response variable and their last daughters' lifespan as explanatory variable, or intercept only models (null model) as comparative models. 
675 Model selection (Table S6) does not allow distinguishing between the two alternative models and we ca nnot completely rule out that the longer-lived mothers produce slightly shorter-lived late daughters (Fig

677 . 2G). In any case, the effect size is weak and such a negative correlation would suggest that longer-live $678 \mathrm{~d}$ mothers accumulate more damage that is then partly transmitted to their late daughter. Similar results 679 hold for late daughters to second generation late daughters (Fig. S8). We cannot completely rule out a w 680 eak tendency that long-lived (late daughter) mothers produce last daughters (second generation late dau 681 ghters) that live slightly shorter (Table S6). In any case, our simulations with a low fixed transmission r 682 ate of $7 \%$ of damage transmitted between mother and daughters would lead to much higher correlation i $683 \mathrm{n}$ lifespan as observed (Fig. 3E main text).

684

685

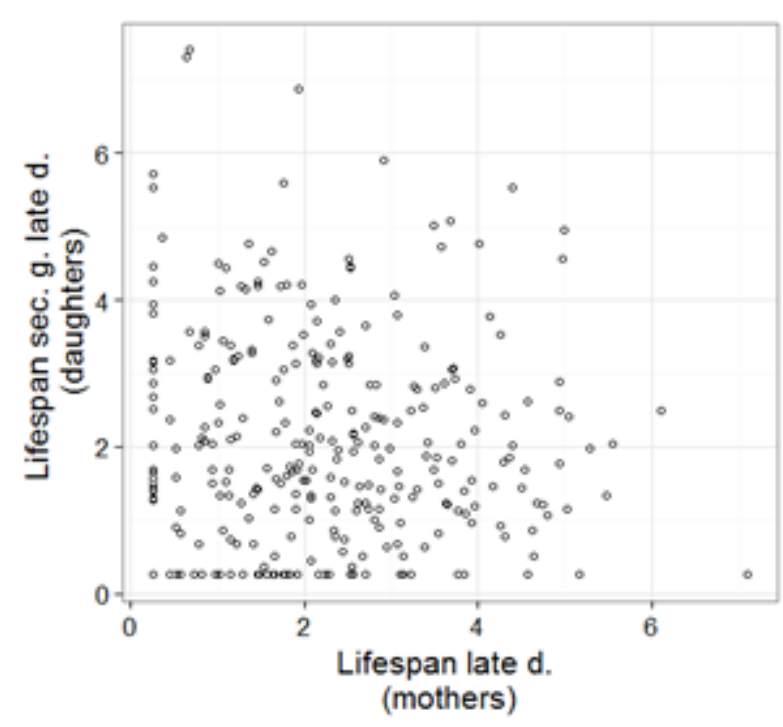

686 Fig. S8: Correlation between lifespan (Square root-transformed) of the 298 late daughters [mothers] 687 versus the lifespan of their last daughter cell, the second generation late daughters (Table S6).

689 Table S6: Model selection for correlation between lifespan (Square root transformed, Gaussian error) Model 


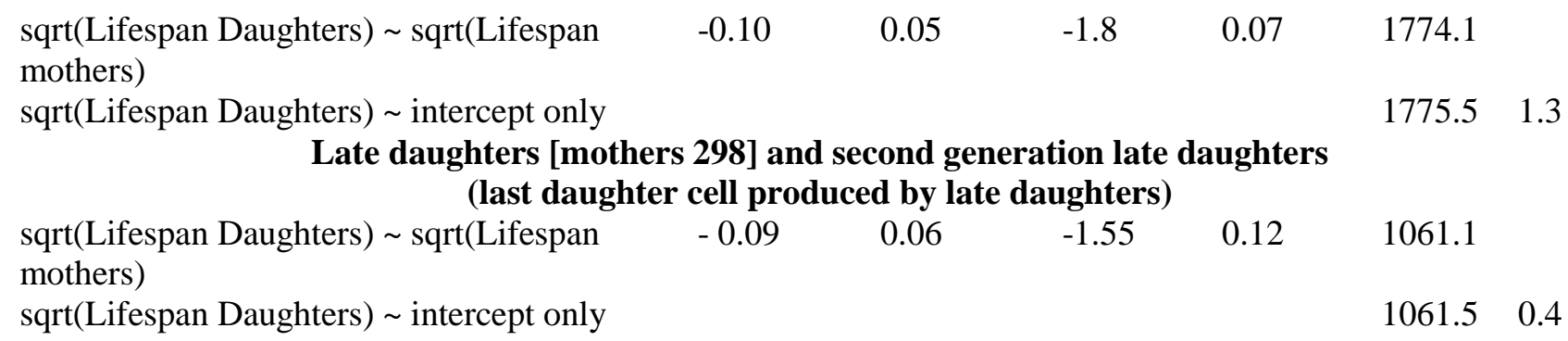

\section{Lifespan and lifetime reproductive success: reproductive and chronological aging}

692 Previous aging studies on E. coli (Stewart et al. 2005, Wang et al. 2010) and other bacteria (Ackermann 693 et al. 2003) or many yeast studies (Denoth Lippuner et al. 2014) have focused on replicative aging and 694 patterns have been described for time measured in number of divisions. Such age measures can be 695 difficult to compare directly to chronological age if the time between divisions varies. Nonetheless, we 696 detected a strong correlation between lifespan and the lifetime reproductive success (number of divisions 697 an individual undergoes throughout its life), but there remains some variation, particularly for longer 698 lived individuals (Fig. S4).

699
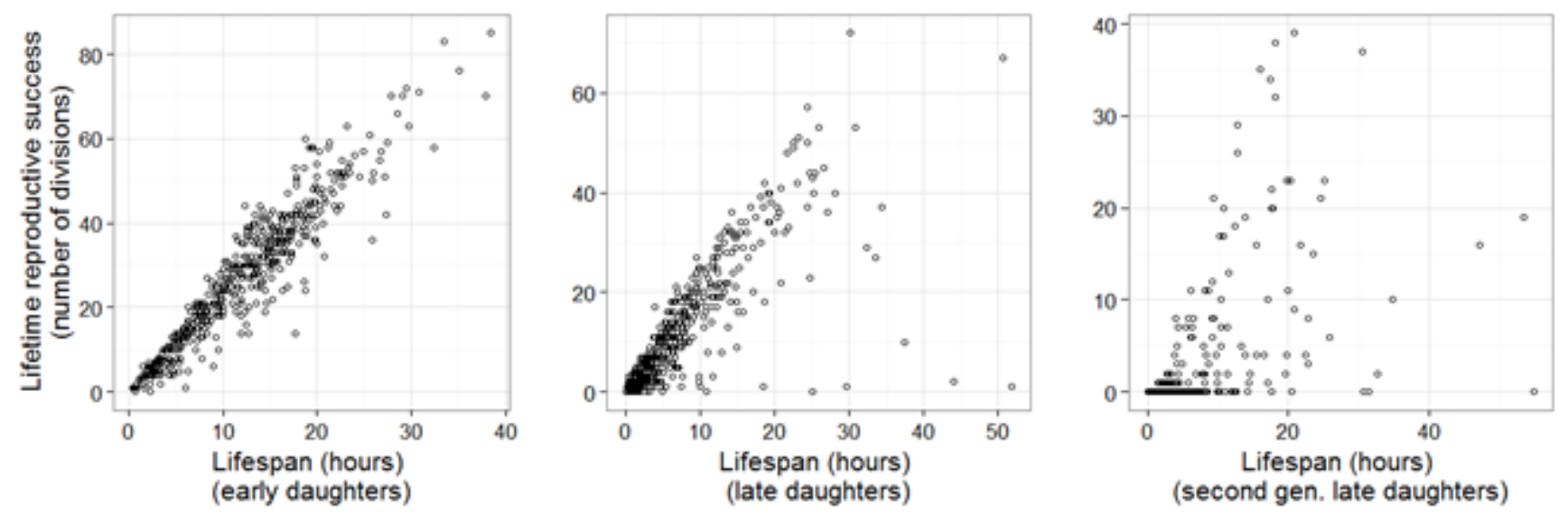

700 Fig. S4: Correlation between lifespan and the lifetime reproductive success (cumulative number of 701 divisions an individual undergoes throughout its life) for left panel) the 516 early daughters, mid panel) 702 the 516 late daughters, and right panel) the 298 second generation late daughters. 


\section{Division rate}

705 We fitted linear models on hourly average division rates (at the cellular level) as response variable and 706 age as explanatory variable. A model with a linear and quadratic term was best supported for the early

707 (Fig. 2A), late (Fig. 2B) and second generation late (Fig. S3A) daughters (Table S2).

708

709 Table S2: Model selection division rate

\begin{tabular}{|c|c|c|c|c|}
\hline Model & $\begin{array}{r}\text { Slope } \pm \text { Std. Error } \\
\text { Earl }\end{array}$ & $\begin{array}{l}\text { Quadratic } \\
\text { term } \pm \text { StdE } \\
\text { ughters }\end{array}$ & AIC & $\triangle \mathrm{AIC}$ \\
\hline $\begin{array}{l}\text { Divison rate -intercept } \\
\text { only }\end{array}$ & & & 19663 & 24 \\
\hline Divison rate age & $-0.011 \pm 0.002$ & & 19642 & 21 \\
\hline $\begin{array}{l}\text { Divison rate } \\
\sim \text { age+age }{ }^{\wedge} 2\end{array}$ & $0.005 \pm 0.0078$ & $-0.0007 \pm 0.0003$ & 19638 & \\
\hline \multicolumn{5}{|c|}{ Late daughters } \\
\hline $\begin{array}{l}\text { Divison rate -intercept } \\
\text { only }\end{array}$ & & & 12432 & 24 \\
\hline Divison rate -age & $-0.009 \pm 0.004$ & & 12428 & 20 \\
\hline $\begin{array}{l}\text { Divison rate } \\
\sim \text { age+age }{ }^{\wedge} 2\end{array}$ & $0.039 \pm 0.011$ & $-0.002 \pm 0.0005$ & 12408 & \\
\hline \multicolumn{5}{|c|}{ Second generation late daughters } \\
\hline $\begin{array}{l}\text { Divison rate -intercept } \\
\text { only }\end{array}$ & & & 5494 & 63.3 \\
\hline Divison rate $\sim$ age & $0.009 \pm 0.004$ & & 5490.3 & 59.6 \\
\hline $\begin{array}{l}\text { Divison rate } \\
\text {-age+age }{ }^{\wedge} 2\end{array}$ & $0.090 \pm 0.011$ & $-0.004 \pm 0.0004$ & 5430.7 & \\
\hline
\end{tabular}

711 Mortality rate

712 We fitted Gamma-Gompertz-Makeham related models to the survival data. GGM models were tested for 713 assumptions among parameters, for instance, whether shape and scale parameters are independent or

714 defined by a rate parameter. For late (Fig. 2D) and second generation late daughters (Fig. S3B), we 715 optimized the scaling of the mother to daughter state relationship (transmission factor) based on the age 
at death state distribution of early daughters (or late daughters for second generation late daughters) (Fig.

717 S10). We assumed that accumulation of damage, which is the stage transition probabilities did not differ

718 among early, late and second generation late daughters. We also assumed that the effect of the stage was

719 not different for early, late or second-generation late daughters. These assumptions allowed us to use the

720 translated GGM estimates of the early daughters also for late and second generation late daughters.

721

722

723

724 725

726

727

728

729

730
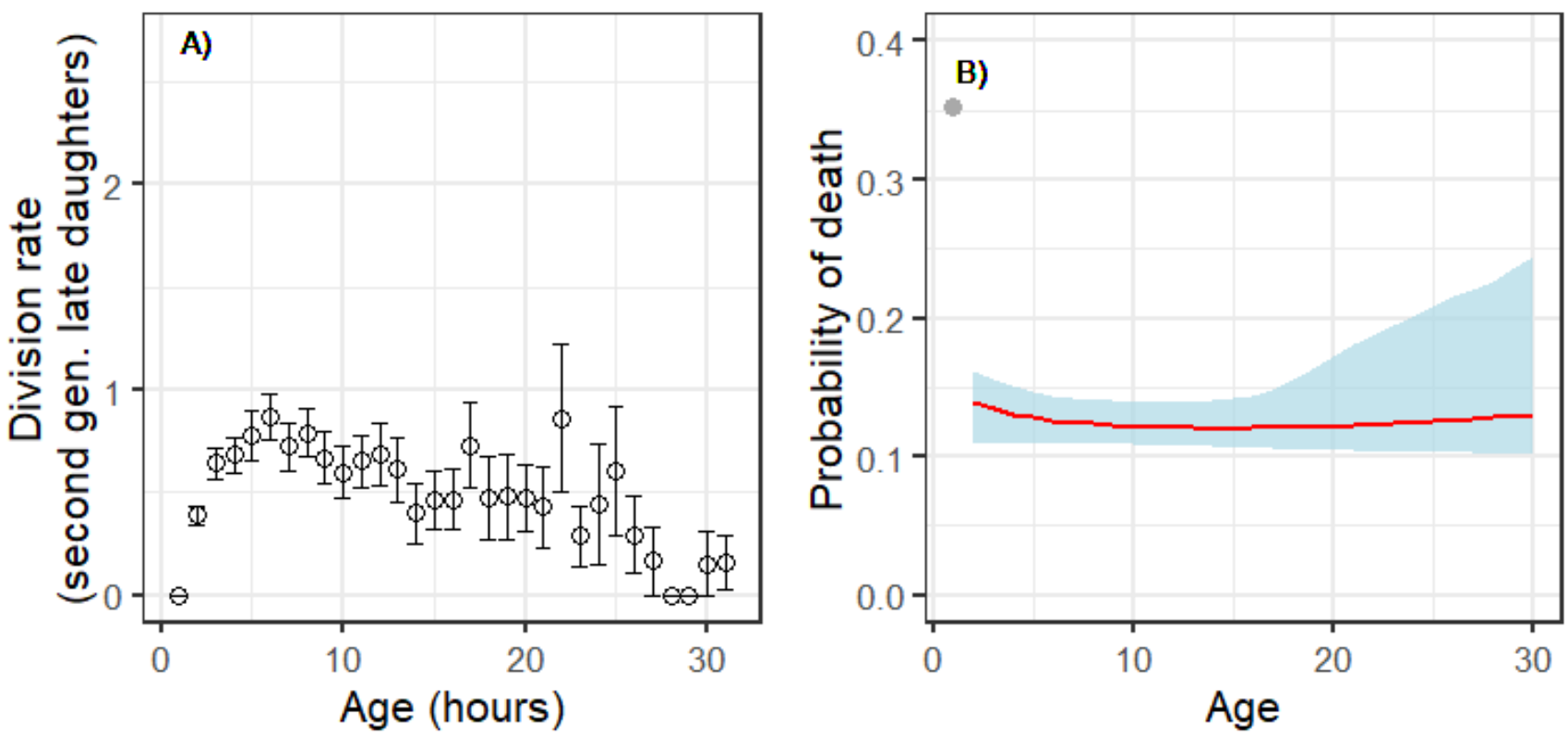

Fig. S3: Age-specific division rate (number of divisions per hour) (A) and age-specific hourly mortality rate (B) of the 298 second generation late daughters. For (A) means \pm standard errors are plotted, for (B) fitted regression $\pm 95 \%$ Confidence Intervals are plotted. Associated model selection results are presented in Table S2.

\section{Difference in mortality rates at old ages for early and late daughters}

The late age mortality plateau detected in early daughters (Fig. 2C) and the flat mortality hazard pattern of the late daughters might indicate similar mortality rates at older ages ( $>19$ hours). To test for this we compared models that distinguished between early and late daughters but only included cells that 
731 survived to an age of at least 20 hours. Late daughter cells have lower mortality rates (qx=0.1248 \pm 0.0191

732 mean \pm Std.Err.) than early daughters at old ages ( $q x=0.2005 \pm 0.0168$ mean \pm Std.Err.) (Table S3) . This

733 result comprises 73 early daughters and 41 late daughters that lived longer than 19 hours.

734 Table S3: Model selection for age-specific mortality rates $\left(q_{\mathrm{x}}\right)$ of old $(>19 \mathrm{~h})$ early daughters and old 735 (>19h) late daughters, based on binomial models.

\begin{tabular}{|c|c|c|c|}
\hline $\begin{array}{l}\text { Model } \\
\text { Mortality } \text { intercept only }\end{array}$ & Estimate \pm Std. Error & Quadratic term & $\begin{array}{l}\text { AIC } \\
95.79\end{array}$ \\
\hline Mortality cell type & $-0.591 \pm 0.211$ & & 89.7 \\
\hline
\end{tabular}

736

\section{Cell elongation rates}

738 We illustrate findings for cell elongation rates for early, late and second generation late daughters (Fig. 739 S2). When comparing linear models, the best fitted model for all three cell types was a model with a 740 linear and quadratic term, though the curvature was minimal for early daughters (Table S1). Early and 741 late daughters showing a decline in cell elongation rate with age, while second generation late daughters 742 patterns are less clear. 

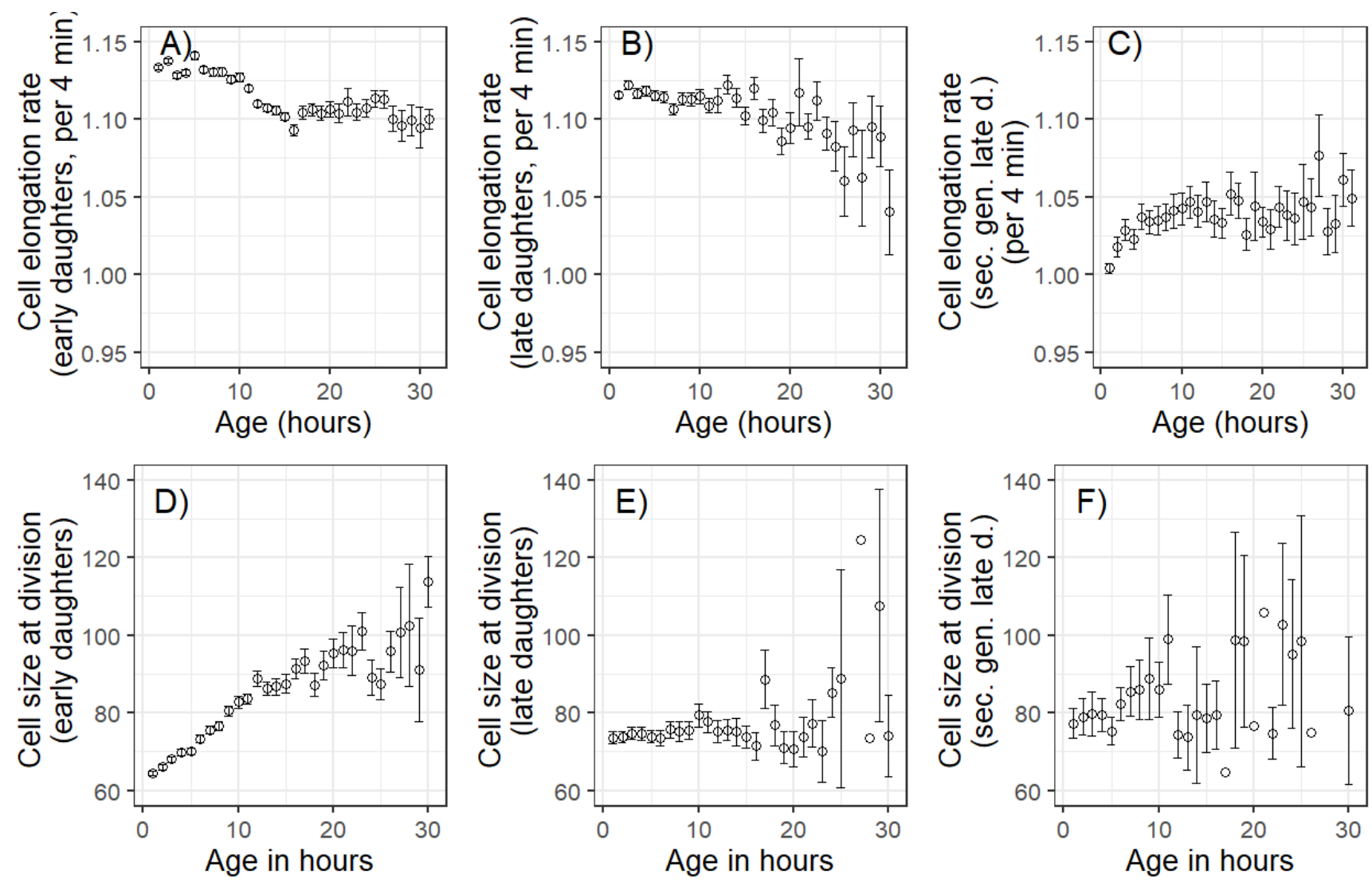

745 Fig. S2 Cell elongation and cell size:

746 A-C) Age-specific cell elongation rate (fractional elongation) and D-F) cell size at division (in pixels,

747 1pixel=0.064 $\mu \mathrm{m}$ ) of the A\&D) 516 early daughter cells, B\&E) 516 late daughter cells, and C\&F) 298

748 second generation late daughter cells. Hourly means \pm standard errors are plotted. Associated model

749 comparisons are presented in Table S1).

750

751 Table S1: Model selection for age-specific cell elongation rates and cell size at division.

\begin{tabular}{|c|c|c|c|c|}
\hline \multicolumn{5}{|c|}{ Cell elongation rates } \\
\hline Model & $\begin{array}{r}\text { Slope } \pm \text { Std. Error } \\
\text { Earl }\end{array}$ & $\begin{array}{l}\text { Quadratic term } \\
\text { ighters }\end{array}$ & AIC & $\triangle \mathrm{AIC}$ \\
\hline $\begin{array}{l}\text { Elongation rate -interc. } \\
\text { only }\end{array}$ & & & -21415 & 407 \\
\hline Elongation rate -age & $-0.002 \pm 0.0001$ & & -21820 & 2 \\
\hline $\begin{array}{l}\text { Elongation rate } \\
\sim \text { age }+a^{\wedge} e^{\wedge 2}\end{array}$ & $-0.002 \pm 0.0002$ & $-0.00002 \pm 0.00001$ & -21822 & \\
\hline
\end{tabular}

\section{Late daughters}




\begin{tabular}{|c|c|c|c|c|}
\hline $\begin{array}{l}\text { Elongation rate -interc. } \\
\text { only }\end{array}$ & & & -10264 & 45 \\
\hline Elongation rate age & $-0.00102 \pm 0.00016$ & & -10304 & 5 \\
\hline $\begin{array}{l}\text { Elongation rate } \\
\sim \text { age }+ \text { age }{ }^{\wedge} 2\end{array}$ & $0.00013 \pm 0.00047$ & $-0.000052 \pm 0.00002$ & -10309 & \\
\hline \multicolumn{5}{|c|}{ Second generation late daughters } \\
\hline $\begin{array}{l}\text { Elongation rate -interc. } \\
\text { only }\end{array}$ & & & -4042.4 & 32.1 \\
\hline Elongation rate -age & $\begin{array}{l}0.0014735 \pm 0.00029 \\
59\end{array}$ & & -4065.1 & 10.4 \\
\hline $\begin{array}{l}\text { Elongation rate } \\
\sim \text { age }+\operatorname{age}^{\wedge 2}\end{array}$ & $0.00429 \pm 0.00088$ & $-0.00012 \pm 0.000035$ & -4074.5 & \\
\hline \multicolumn{5}{|c|}{ Cell size at division } \\
\hline Model & $\begin{array}{r}\text { Slope } \pm S t d . \text { Error } \\
\text { Early }\end{array}$ & $\begin{array}{l}\text { Quadratic term } \\
\text { Ighters }\end{array}$ & AIC & $\triangle \mathrm{AIC}$ \\
\hline $\begin{array}{l}\text { Size at division -interc. } \\
\text { only }\end{array}$ & & & 53741 & 1378 \\
\hline Size at division - age & $1.72 \pm 0.04$ & & 52431 & 68 \\
\hline $\begin{array}{l}\text { Size at division } \\
\sim \text { age }+\operatorname{age}^{\wedge 2}\end{array}$ & $2.797 \pm 0.135$ & $-0.049 \pm 0.006$ & 52362 & \\
\hline \multicolumn{5}{|c|}{ Late daughters } \\
\hline $\begin{array}{l}\text { Size at division -interc. } \\
\text { only }\end{array}$ & & & 28592 & 101 \\
\hline Size at division age & $0.75 \pm 0.09$ & & 28525 & 34 \\
\hline $\begin{array}{l}\text { Size at division } \\
\sim a q e+a q e^{\wedge 2}\end{array}$ & $2.204 \pm 0.259$ & $-0.069 \pm 0.012$ & 28491 & \\
\hline \multicolumn{5}{|c|}{ Second generation late daughters } \\
\hline $\begin{array}{l}\text { Size at division -interc. } \\
\text { only }\end{array}$ & & & 8381.7 & 276.2 \\
\hline Size at division -age & $3.036 \pm 0.223$ & & 8215.9 & 110.4 \\
\hline $\begin{array}{l}\text { Size at division } \\
\sim \text { age }+\operatorname{age}^{\wedge} 2\end{array}$ & $8.919 \pm 0.576$ & $-0.293 \pm 0.027$ & 8105.5 & \\
\hline
\end{tabular}

\section{Size at division}

754 We illustrate cell size at division for early, late, and second generation late daughters (Fig. S2). When 755 comparing linear models, the best fitted model for all three cell types was a model with a linear and 756 quadratic term, though the curvature was minimal for early and late daughters (Table S1). Early daughters 757 showed clear senescence in cell elongation rate, late daughters showed weaker senescence in cell 758 elongation rate, and patterns for second-generation late daughters are less clear. 


\section{Reverse time analyses: Hours before death}

761 Chronological aging follows individuals from birth to death and time counted starts at birth. Various

762 hypotheses about the evolution of life histories, aging and senescence, in particular, terminal investment 763 strategies (Williams 1966, Clutton-Brock 1984, Charlesworth 1994), assume that individuals can sense 764 that they are approaching death and that an optimal strategy for an individual might be to invest remaining 765 resources into reproduction rather than maintenance when approaching immediate death. Such terminal 766 investment has also been labeled as terminal illness, last year effect, or similar terms, and have been studied in a broad range of taxa, also using reverse time analyses (Coulson and Fairweather 2001). As

Fig. 2 \& Fig. 3 in the main text illustrates, age at death is highly variable, for that chronological senescence patterns and terminal investment strategies might provide very different insights. We investigated whether patterns in reproduction (division rate), cell elongation rate, or size at division differ that the last hour before death would show significant changes compared to the more spread out chronological senescence patterns (Fig. 2, Fig. S3 \& S4). In general, there seems to be little evidence that

774 terminal investment is happening to a larger degree, at least such strategies if existent, do not show larger 775 effects compared to chronological aging and classical senescence (Fig. S5 \& S6, Table S4 \& S5).

776

\section{Division rate for hours before death}

778 When individual cells approach death, their division rates decrease (Fig. S5), and these senescence 779 patterns are similar compared to the (chronological) senescence patterns (Fig. 2A, B) (Table S4 \& Table 780 S2). 

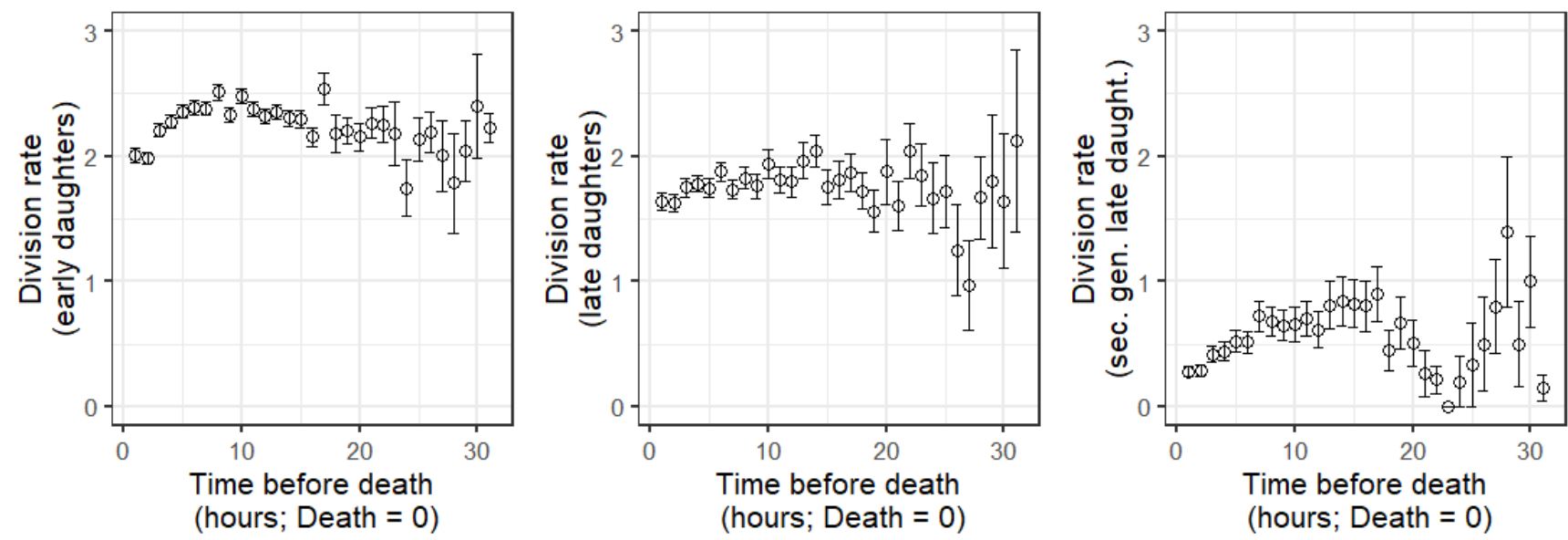

783 Fig. S5: Hourly division rate for hours before death [remaining life time] (death = time 0) for left panel

784 the 516 early daughters cells, mid panel the 516 late daughters, and right panel the 298 second generation

785 daughters. Means \pm standard errors are plotted. Associated model selection is shown in Table S4.

Table S4: Model selection for division rate in reverse time (starting with age at death=0).

Model

Division rate -interc. only

Division rate $\sim$ reverse

time

Division rate $\sim$ reverse time + reverse time $\wedge \mathbf{2}$

Division rate -interc. only

Division rate $\sim$ reverse

time

Division rate $\sim$ reverse

time + reverse time $\wedge^{\wedge} \mathbf{2}$

Division rate -interc. only

Division rate $\sim$ reverse

time

Division rate $\sim$ reverse

time + reverse time $\wedge 2$
Slope \pm Std. Error

\section{Early daughters}

$0.006 \pm 0.002$

$0.062 \pm 0.0070$

$-0.002 \pm 0.0003$

\section{Late daughters}

$0.006 \pm 0.003$

121718

$12170 \quad 7$

$0.036 \pm 0.017$

$-0.001 \pm 0.0004$

12163

Second generation daughters

$0.015 \pm 0.003$

$0.069 \pm 0.017$

$-0.002 \pm 0.0004$

$5474.5 \quad 47.8$

5456

29.3 
790 The cell elongation rate does not drastically fall off when approaching death (no initial steep increase in

791 cell elongation rate close to the time of death) (Fig. S6, Table S5). The senescence pattern is, at least for 792 early and late daughters, less pronounced compared to that observed for chronological senescence (Fig. 793 S2). Second generation daughters seem to reduce cell growth rates before death more substantially, but 794 this reduction already starts a few hours before death and is not in line with terminal investment theories.
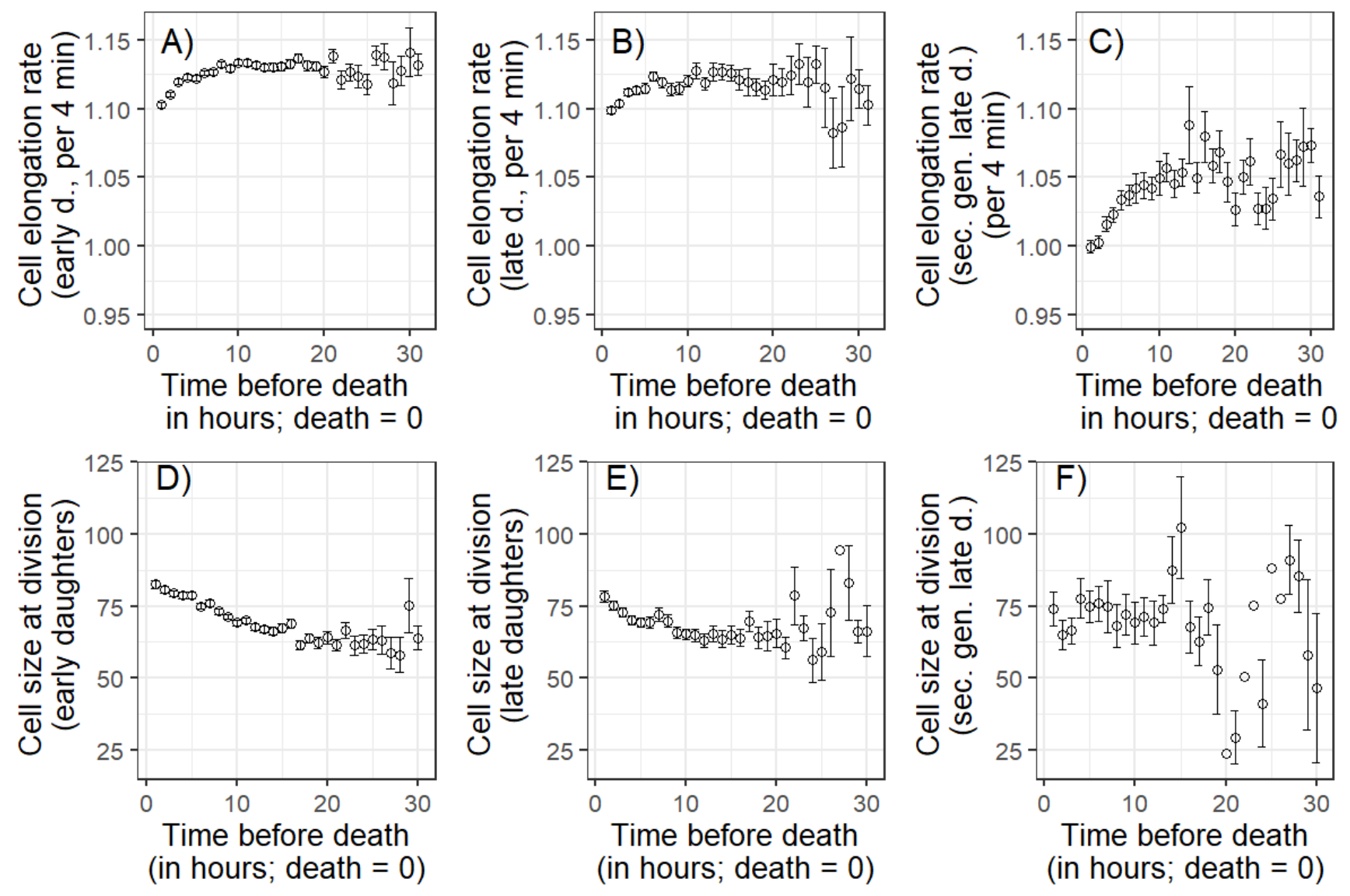

796

Fig. S6: (Fractional) Cell elongation rate and cell size at division (in pixels, 1pixel=0.064 $\mu \mathrm{m}$ ) for hours

before death (death = time 0) for A \& D) the 516 early daughters, B \& E) the 516 late daughters, and C

\& F) the 298 second generation late daughters. Means \pm standard errors are plotted. Associated model selection is shown in Table S5. 
801 Table S5: Model selection for cell elongation and cell size at division rate in reverse time (starting with

802 age at death=0).

Model

Elongation rate -interc.

only

Elongation rate - reverse

time

Elongation rate

reverse time + reverse

time $\wedge^{\wedge}$

Elongation rate $\sim$ interc.

only

Elongation rate -reverse

time

Elongation rate

reverse time + reverse

time $\wedge^{\wedge}$

Elongation rate -interc.

only

Elongation rate - reverse

time

Elongation rate -

reverse time + reverse

time $\wedge 2$

Size at division -interc.

only

Size at division reverse

time

Size at division

reverse time + reverse

time $\wedge 2$

Size at division -interc.

only

Size at division reverse

time

Size at division

reverse time + reverse

time $\wedge 2$

Size at division -interc.

only
Cell elongation (reverse time)

Slope \pm Std. Error Quadratic term

Early daughters

$0.001 \pm 0.00009$

AIC $\triangle \mathrm{AIC}$

$-22138250$

$-22288100$

$0.004 \pm 0.0003 \quad-0.0001 \pm 0.00001$

$-22388$

Late daughters

$0.001 \pm 0.0001$

$-1052965$

$-1056133$

$\begin{array}{lll}0.003 \pm 0.0004 & -0.0001 \pm 0.00002 & -10594\end{array}$

Second generation late daughters

$0.003 \pm 0.0003$

$-4082.8122 .1$

$-4167.5 \quad 37.4$

$0.008 \pm 0.0009$

$-0.0002 \pm 0.00003$

$-4204.9$

Cell size at division (reverse time) Early daughters

$-0.952 \pm 0.0486$

53679386

532996

$\begin{array}{lll}-1.337 \pm 0.145 & 0.018 \pm 0.006 & 53293\end{array}$

Late daughters

$-0.438 \pm 0.088$

$28629 \quad 26$

$28606 \quad 3$

$\begin{array}{lll}-0.990 \pm 0.258 & 0.026 \pm 0.011 & 28603\end{array}$

Second generation late daughters

$8182.3 \quad 54.9$ 
Size at division $\sim$ reverse time

Size at division reverse time + reverse time $\wedge 2$
$1.254 \pm 0.236$

$4.531 \pm 0.627$
8156.6

$-0.158 \pm 0.028$
8127.4
29.2

\section{Cell size at division for hours before death}

805 Cell size at division increased with chronological age, but mainly for early daughters (Fig. S2). One 806 mechanism that might lead to such an increase in cell size at division is the formation of filamentous 807 cells: cells that do continue to elongate but stop dividing. Such filamentation is usually seen as a stress 808 response and increases mortality of the cell (Mattick et al. 2003, Wang et al. 2010). Previous studies 809 (conducted under different media and temperature than ours) have reported higher filamentation rates 810 with increasing age (Wang et al. 2010). Under our growth conditions, cells that entered a filamentation 811 stage rarely recovered from that stage and did not return to a regular dividing smaller cell, though they 812 not always stalled dividing. To test if such filamentous cells drive the chronological senescence pattern 813 in size at division, we investigated the cell size for the hours before death. For early and late daughters, 814 we detected an increase in cell size at division before death, but this increase is already initiated hours 815 before the actual death and not a final increase in size due to filamentation (Fig. S6, Table S5).

\section{Random deterioration model}

818 Our random deterioration model builds on a LeBras type cascading failure model(Le Bras 1976). In such 819 discrete state models, individuals increase from a current state $i$ to a state $i+1$ at a rate proportional to $i$, 820 and mortality increases proportionally to state $i$. We can think of such states as accumulating damage, or 821 model the reverse process, where an individual starts with some vitality and this vitality then decreases 822 with increasing state $i$ (random deterioration) (Weitz and Fraser 2001). In this study, we assume 
823 accumulating damage that stands symbolically for an unknown aging factor. These random deterioration

824

825

826

827

840 We then transformed these estimates of the GGM model to a random deterioration model according to

841 Yashin et al. (Yashin et al. 1994), making use of mathematical similarities between the GGM and random

842 deterioration models.

843 Let $t(i)=\lambda_{0}+i \lambda, m(i)=\mu_{0}+i \mu$ (for $i$ greater than or equal to 0 ) denote the state transition rate and the

844 death rate, respectively.

models are mathematically closely linked to demographic frailty models that do not assume a stochastic stage progression among individuals, but rather heterogeneity of individual is defined by fixed frailty at birth (Yashin et al. 1994). Such frailty, an age independent mortality that follows a gamma distribution (Missov and Vaupel 2015), can be added to the classical models in demography, a Gompertz- model, which describes an age-dependent exponential increase in mortality. These models can also be extended by a Makeham term, an age-independent baseline mortality, which further improves model fit, at least for humans and various other complex species.

We first estimated parameters of such a Gamma-Gompertz-Makeham (GGM) model, Zae $e^{b x}+c$ fitted to the survival data of the early daughters. $Z$ is the random frailty variable that follows a gamma distribution across individuals. The distribution has a mean 1 and variance of $\sigma^{2}$. This definition of a gamma distribution with a mean of 1 implies that we have to set the scale parameter equal to the shape parameter of the gamma distribution. The rate of aging, $b$, is the same for all individuals, $x$ determines the age in hours, $a$ describes the baseline mortality of the Gompertz part of the model, and $c$ describes the Makeham term, the age independent mortality.

$$
\mu_{0}=\mathrm{a}+\mathrm{c} \text {, the death rate at birth i.e. state } i=0 \text {, }
$$



$\mu=\sigma^{2} a$, the change in the death rate at state $i$, for $i>0$

850 Resulting estimates for early daughters were $\mu_{0}=0.056, \lambda_{0}=0.178, \lambda=0.598$, and $\mu=0.00023$.

851 Using these estimates for early daughters, we estimated the probability of observing an individual at state $852 i$ at age $x, P(x, i)$,

853

$$
P_{i}(x)= \begin{cases}P_{0}(0) e^{-\left(\lambda_{0}+\mu_{0}\right) x}, & i=0 \\ \frac{P_{0}(x)}{i !}\left[\frac{\lambda-\lambda e^{-(\lambda+\mu) x}}{\lambda+\mu}\right] \prod \prod_{k=1}^{i}\left(\frac{\lambda_{0}}{\lambda}+(k-1)\right), & i>0\end{cases}
$$

854 We then built a matrix of $P(x, i)$ for which we limited the age, $x$, between 0 and 31 and the states were 855 limited between 0 and 5000. The column sum of this matrix equals the survival probability at any given 856 age $x$ to age $x+1$.

\section{Microsimulations of the random deterioration model}

858 In order to simulate the (damage) state at death distribution we used a microsimulation. As a step-by859 step calculation would take too long, we modelled the state at death distribution by drawing of a Poisson 860 distribution with a rate of $\frac{1}{\mu_{0}+\hat{\imath} \mu}$, where $\hat{\imath}=i+\frac{1}{\mu_{0}+i \mu}$. In doing so, we estimated the expected state at 861 death, rather than tracking individuals transitioning through damage states and their associated mortality 862 risk as a step-by-step calculation would do. A step-by-step simulation would take a long time because 863 individuals can easily end up in very high damage states (very long tailed distributions). 
866 For the late daughters, we used the same state transition and survival parameters $\left(\mu_{0}=0.056, \lambda_{0}=0.178\right.$, $867 \lambda=0.598$, and $\mu=0.00023$ ) that we estimated for the early daughters. We did so because early and 868 late daughter cells should biologically not be fundamentally different - aside of starting with different damage levels at birth. They should accumulate damage at the same rate and should experience mortality risk just based on their damage state. We assumed that the late daughters are born at the same stage, or a

871

874 Where subscript late and early denotes late and early daughter estimates and $s \in(0,1)$ and $\operatorname{nint}($.$) is$ 875 876

scaled version of the same stage, in which the mothers died, therefore let

$$
\begin{aligned}
& \lambda_{0, \text { late }}=\operatorname{nint}\left(s\left(\lambda_{0, \text { early }}+i \lambda\right)\right) \\
& \mu_{0, \text { late }}=\operatorname{nint}\left(s\left(\mu_{0, \text { early }}+i \mu\right)\right)
\end{aligned}
$$

the nearest integer function. So based on the estimated damage state distribution of the mothers at death (early daughters), we also knew the damage state distribution at birth of the daughters (late daughters) [see below for optimally scaling this distribution]. Using the state transition probabilities of the early daughters and the probabilities of death, we estimated the survival of the late daughters. We also estimated the survival functions of cells that did not change their damage state with age. Each grey line in main text Fig. 3, describes such a fixed damage class survival function.

In order to determine the transmission fraction of accumulated damage between mothers (early daughters) and daughters (late daughters), we optimized the scaling of the mother state at death distribution (fraction of mother-daughter transmission) to the daughter age at birth distribution. The optimization was based on observed survival of the late daughters, by minimizing the squared deviations from the observed survival function. This optimal scaling factor was estimated at $7 \%$.

Similar to the late daughters, we simulated the damage state distribution at birth, the damage state distribution at death, and calculated the matrix $P(x, i)$ for the second generation late daughters. 
888

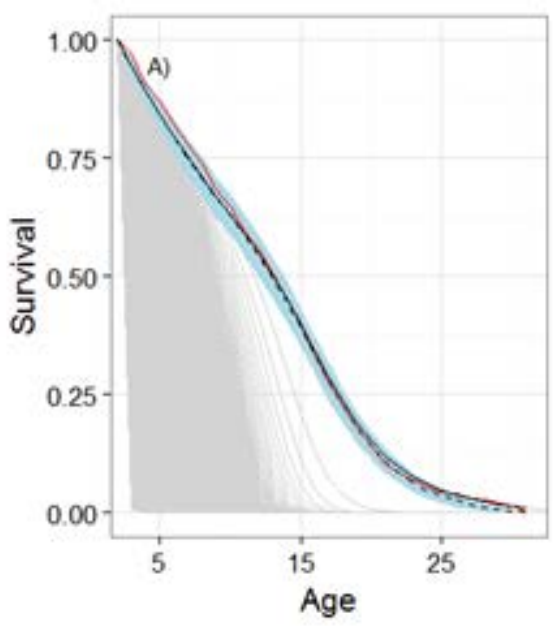

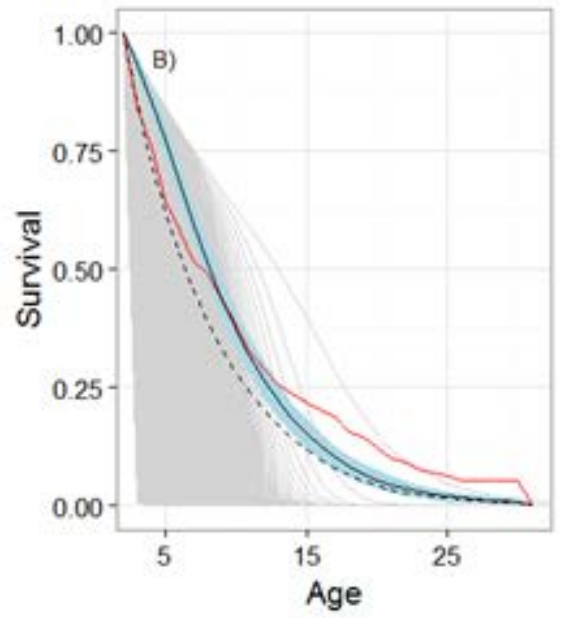

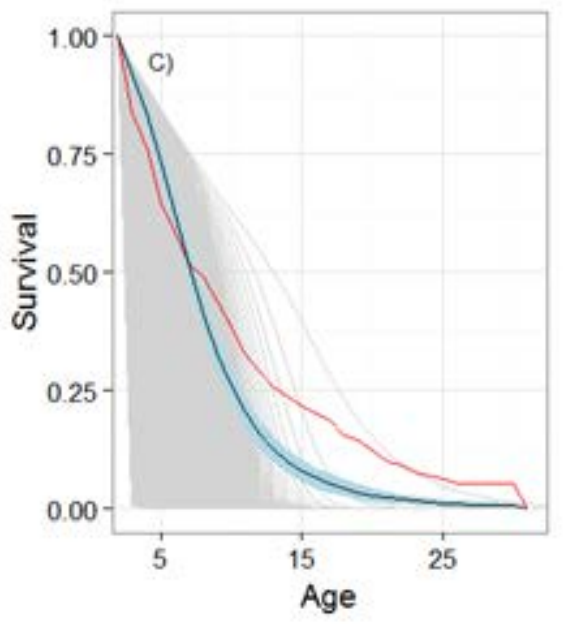

889 Fig. S9: Observed population level survival curve (red line), GGM simulated survival curve with 890 symmetric damage transmission (hatched black line) as well as GGM simulated survival curve with 891 asymmetric damage transmission of 7\% (solid black line $\pm 95 \%$ CI in blue) for early (A), late (B) and 892 second generation late daughters (C). Graphs A \& B are identical to Fig. 1C \& D. Thin grey lines in 893 depict expected survival curve of cells with different fixed damage state. That is, for instance, the 894 outermost thin grey line in B depicts the survivorship curve of a hypothetical cohort that starts without 895 damage and never accumulates any damage. The most left survival curve illustrates the low survival of 896 cells that were born with maximum damage level of 5000. 


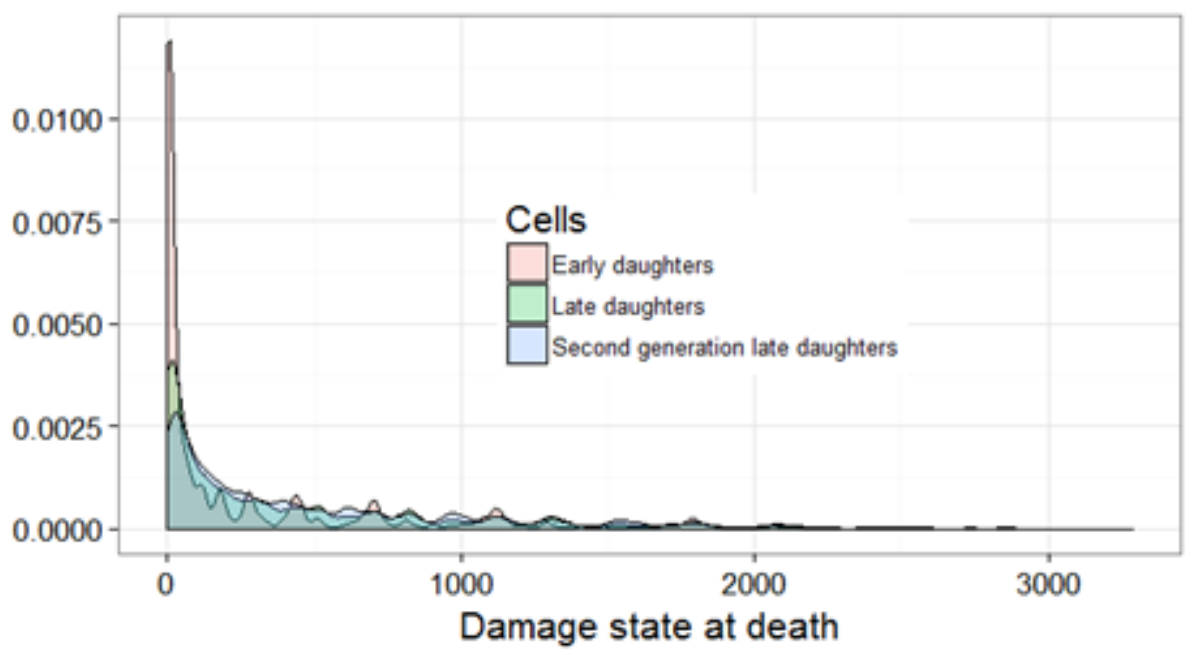

899 Fig. S10: Density distribution of damage state at death of early daughters, late daughters, and second
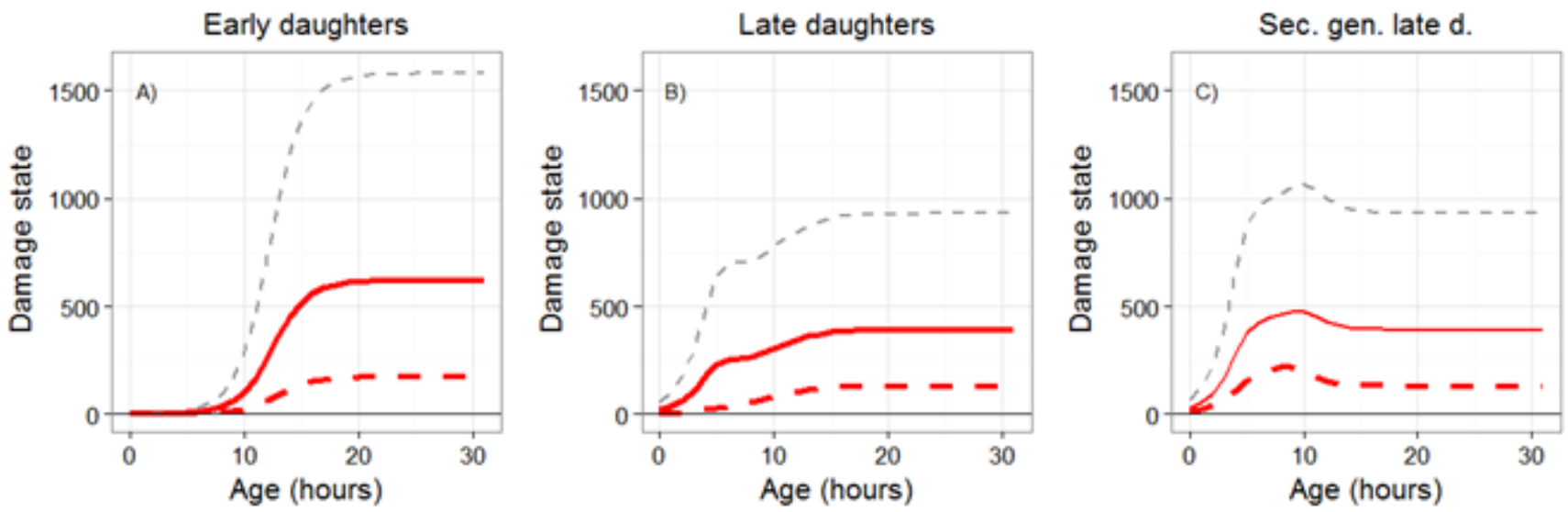

904 Fig. S11: GGM model results: mean (red solid line, + SD grey hatched lines) and median (red hatched 905 line) damage state with increasing age for early daughter cells (A), and late daughter cells (B) and second 906 generation late daughter cells (C). Panel A \& B are identical to Panel Fig. 1A, B. 


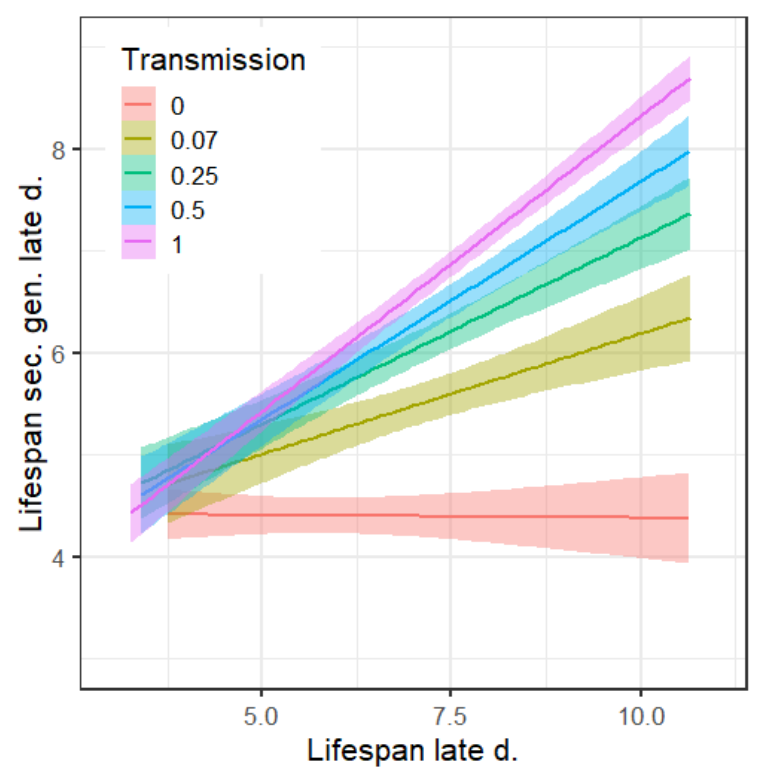

909 Fig. S12: Lifespan (square root transformed) of simulated 516 late daughter cells (mothers) versus the 910 lifespan of their simulated last daughter cell (second generation late daughter cells) with different levels 911 of mother to daughter damage transmission. Scenarios include the optimized fixed transmission level at 9120.07 (forest green), a scenario for perfect rejuventation, i.e. 0 transmission (red), 0.25 transmission 913 (green), 0.5 transmission, i.e. symmetric (equal) transmission between mother and daughter (blue), and 914 transmission of all accumulated damage to the daughter (1) (pink).CI are shown for each correlation in 915 lifespan between mothers and daughters as shaded areas.

\section{References}

918 Ackermann, M. 2015. A functional perspective on phenotypic heterogeneity in microorganisms. - Nat. Rev. Microbiol. 13: 497-508.

920 Ackermann, M. et al. 2003. Senescence in a bacterium with asymmetric division. - Science 300: 1920.

921 Ackermann, M. et al. 2007. On the evolutionary origin of aging. - Aging Cell 6: 235-44. 
922 Aoki, S. K. et al. 2005. Contact-Dependent Inhibition of Growth in Escherichia coli. - Science (80-. ). 923 in press.

924 Balázsi, G. et al. 2011. Cellular decision making and biological noise: from microbes to mammals. $925 \quad$ Cell 144: 910-925.

926 Blattner, F. R. 1997. The Complete Genome Sequence of Escherichia coli K-12. - Science (80-. ). 277: $927 \quad 1453-1462$.

928 Brillinger, D. R. 1986. A Biometrics Invited Paper with Discussion: The Natural Variability of Vital 929 Rates and Associated Statistics. - Biometrics 42: 693.

930 Burnham, K. and Anderson, D. R. 1998. Model selection and multimodel inference : a practical 931 information-theoretic approach. - Springer.

932 Caswell, H. 2001. Matrix population models: construction, analysis, and interpretation. - Sinauer $933 \quad$ Associates.

934 Charlesworth, B. 1994. Evolution in age-structured populations. - Cambridge University Press. 935 Clutton-Brock, T. 1984. Reproductive effort and terminal investment in iteroparous animals. - Am. $936 \quad$ Nat. 123: 212-229. 937 Coquel, A.-S. et al. 2013. Localization of protein aggregation in Escherichia coli is governed by 938 diffusion and nucleoid macromolecular crowding effect. - PLoS Comput. Biol. 9: e1003038. 939 Coulson, J. C. and Fairweather, J. A. 2001. Reduced reproductive performance prior to death in the $940 \quad$ Black-legged Kittiwake: senescence or terminal illness? - J. Avian Biol. 32: 146-152.

941 Davidson, C. J. and Surette, M. G. 2008. Individuality in Bacteria. - Annu. Rev. Genet. 42: $253-268$. 942 Denoth Lippuner, A. et al. 2014. Budding yeast as a model organism to study the effects of age. 943 FEMS Microbiol. Rev. 38: 300-25.

944 Elowitz, M. B. et al. 2002. Stochastic gene expression in a single cell. - Science (80-. ). 297: 1183- 
1186.

946 Evans, S. N. and Steinsaltz, D. 2007. Damage segregation at fissioning may increase growth rates: a superprocess model. - Theor. Popul. Biol. 71: 473-90.

948 Finch, C. and Kirkwood, T. B. 2000. Chance, Development, and Aging. - Oxford University Press. 949 Gangan, M. S. and Athale, C. A. 2017. Threshold effect of growth rate on population variability of $950 \quad$ Escherichia coli cell lengths. - R. Soc. open Sci. 4: 160417.

951 Gasset-Rosa, F. et al. 2014. Direct assessment in bacteria of prionoid propagation and phenotype 952 selection by Hsp70 chaperone. - Mol. Microbiol. 91: 1070-87.

953 Hamilton, W. D. 1966. The moulding of senescence by natural selection. - J. Theor. Biol. 12: 12-45. 954 Jagers, P. 1978. Balanced exponential growth: what does it mean and when is it there? - In: 955 Biomathematics and cell kinetics. Elsevier, pp. 21-29. 956 Johnson, L. R. and Mangel, M. 2006. Life histories and the evolution of aging in bacteria and other 957 single-celled organisms. - Mech. Ageing Dev. 127: 786-93.

958 Jones, O. R. et al. 2014. Diversity of ageing across the tree of life. - Nature 505: 169-73.

959 Jouvet, L. et al. 2017. Demographic variability and heterogeneity among individuals within and among $960 \quad$ clonal bacteria strains. - Oikos in press.

961 Kærn, M. et al. 2005. Stochasticity in gene expression: from theories to phenotypes. - Nat. Rev. Genet. $962 \quad 6: 451-464$.

963 Kennedy, B. K. 1994. Daughter cells of Saccharomyces cerevisiae from old mothers display a reduced 964 life span. - J. Cell Biol. 127: 1985-1993.

965 Kirkwood, T. B. L. 2005. Understanding the odd science of aging. - Cell 120: 437-47.

966 Kirkwood, T. B. L. et al. 2005. What accounts for the wide variation in life span of genetically identical 967 organisms reared in a constant environment? - Mech. Ageing Dev. 126: 439-443. 
968 Klein, J. P. and Moeschberger, M. L. 2003. Survival analysis : techniques for censored and truncated 969 data. - Springer.

970 Le Bras, H. 1976. Lois de mortalité et age limite. - Population (Paris). 33: 655-691.

971 Lindner, A. B. and Demarez, A. 2009. Protein aggregation as a paradigm of aging. - Biochim. Biophys. $972 \quad$ Acta 1790: 980-96.

973 Lindner, A. B. et al. 2008. Asymmetric segregation of protein aggregates is associated with cellular 974 aging and rejuvenation. - Proc. Natl. Acad. Sci. U. S. A. 105: 3076-81.

975 López-Otín, C. et al. 2013. The hallmarks of aging. - Cell 153: 1194-217.

976 Mattick, K. et al. 2003. Morphological changes to Escherichia coli O157:H7, commensal E. coli and 977 Salmonella spp in response to marginal growth conditions, with special reference to mildly 978 stressing temperatures. - Sci. Prog. 86: 103-113.

979 Medawar, P. B. 1952. An unsolved problem of biology. - In: Uniqueness of the Individual. H. K. $980 \quad$ Lewis, in press.

981 Minois, N. et al. 2005. Advances in measuring lifespan in the yeast Saccharomyces cerevisiae. - Proc. $982 \quad$ Natl. Acad. Sci. U. S. A. 102: 402-6.

983 Missov, T. I. and Vaupel, J. W. 2015. Mortality Implications of Mortality Plateaus. - SIAM Rev. 57: $984 \quad 61-70$.

985 Norman, T. M. et al. 2013. Memory and modularity in cell-fate decision making. - Nature 503: 481$986 \quad 486$.

987 Norman, T. M. et al. 2015. Stochastic Switching of Cell Fate in Microbes. - Annu. Rev. Microbiol. 69: $988 \quad 381-403$.

989 R Core Team, R. A. language and environment for statistical computing. 2016. R: A language and 990 environment for statistical computing. (RDC Team, Ed.). - R Found. Stat. Comput. 1: 409. 
991 Scherbov, S. and Ediev, D. 2011. Significance of life table estimates for small populations: Simulationbased study of estimation errors. - Demogr. Res. 24: 527-550.

993 Steiner, U. K. and Tuljapurkar, S. 2012. Neutral theory for life histories and individual variability in 994 fitness components. - Proc. Natl. Acad. Sci. U. S. A. 109: 4684-9.

995 Steinsaltz, D. and Evans, S. N. 2004. Markov mortality models: implications of quasistationarity and 996 varying initial distributions. - Theor. Popul. Biol. 65: 319-37.

997 Stewart, E. J. et al. 2005. Aging and death in an organism that reproduces by morphologically 998 symmetric division. - PLoS Biol. 3: e45.

999 Stroustrup, N. et al. 2016. The temporal scaling of Caenorhabditis elegans ageing. - Nature 530: 103$1000 \quad 107$.

1001 Tyedmers, J. et al. 2010. Cellular strategies for controlling protein aggregation. - Nat. Rev. Mol. cell $1002 \quad$ Biol. 11: 777-788.

1003 Vaupel, J. W. and Yashin, A. I. 1985. Heterogeneity’s Ruses: Some Surprising Effects of Selection on 1004 Population Dynamics. - Am. Stat. 39: 176.

1005 Vaupel, J. W. et al. 1998. Biodemographic Trajectories of Longevity. - Science (80-. ). 280: 855-860.

1006 Vaupel, J. W. et al. 2004. The case for negative senescence. - Theor. Popul. Biol. 65: 339-351.

1007 Vera, M. et al. 2016. Single-Cell and Single-Molecule Analysis of Gene Expression Regulation. 1008 Annu. Rev. Genet. 50: 267-291.

1009 Wachter, K. W. 1999. Evolutionary demographic models for mortality plateaus. - Proc. Natl. Acad. Sci. $101096: 10544-10547$.

1011 Wachter, K. W. et al. 2014. Evolutionary shaping of demographic schedules. - Proc. Natl. Acad. Sci. U. $1012 \quad$ S. A. 111 Suppl: 10846-53.

1013 Wang, P. et al. 2010. Robust growth of Escherichia coli. - Curr. Biol. 20: 1099-103. 
1014 Weitz, J. and Fraser, H. 2001. Explaining mortality rate plateaus. - Proc. Natl. Acad. Sci. U. S. A. 98: $1015 \quad 15383-15386$.

1016 Williams, G. C. 1957. Pleiotropy, Natural Selection, and the Evolution of Senescence. - Evolution (N. 1017 Y). 11: 398.

1018 Williams, G. C. 1966. Adaptation and natural selection : a critique of some current evolutionary 1019 thought. - Princeton Univeristy Press.

1020 Wolf, M. et al. 2007. Life-history trade-offs favour the evolution of animal personalities. - Nature 447: $1021 \quad 581-584$.

1022 Yashin, A. I. et al. 1994. A duality in aging: the equivalence of mortality models based on radically 1023 different concepts. - Mech. Ageing Dev. 74: 1-14. 